\title{
تسوية معدلات الوفاة الخام باستخدام طريقة (Whittaker - Henderson)
}

\author{
د. جيهان مسعد المعداوى \\ مدرس بقسم الاحصاء التطبيقى و التامين \\ كلية التجارة - جامعة المنصورة
}

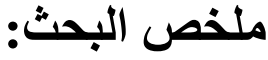

يهذف هذا البحث إلى استخدام طريقة (Whittaker - Henderson) وهى من الطئ الطرق

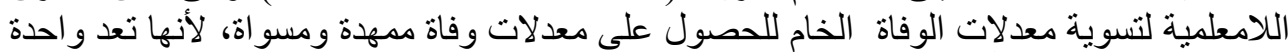

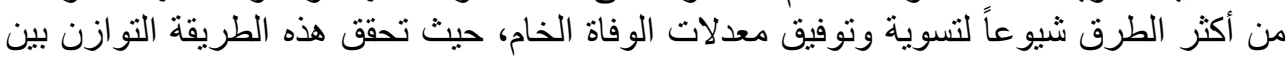

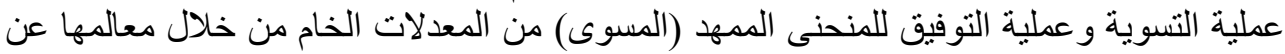

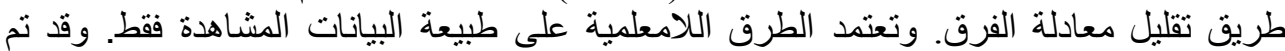
تسوية معدلات الوفاة المركزية الخام للأعمار المفردة بطريقة (Whittaker - Henderson)

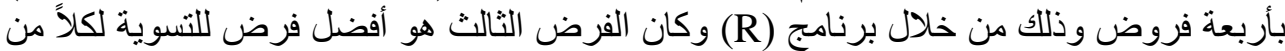

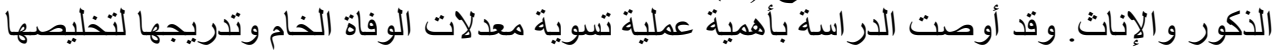

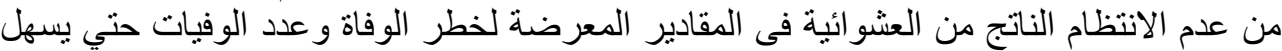

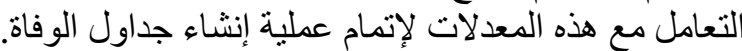

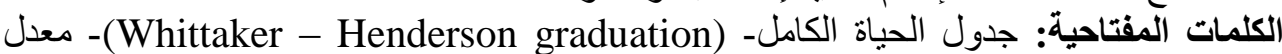
الوفاة المركزى ـ عملية التسوية.
\end{abstract}

\section{Abstract:}

This research aims to use the (Whittaker - Henderson) method, which is an (Nonparametric Method) for graduate, the crude death rates to obtain the smoothness and fitness death rates, because it is one of the most common methods for smoothing the crude death rates, for crude mortality rates. It has the capacity to explicitly balance the smoothness as well as the fitness of the smoothed curve relative to the crude rates through its parameters by minimizing a difference equation. Non-parametric methods depend only on the nature of the observed data. The crude central death rates for the single ages were smoothed and fitted using Whittaker-Henderson graduation technique by four assumptions through the program $(\mathrm{R})$ and the third hypothesis was the best assumption of graduated for both males and females. The study recommended the important of graduation for the crude death rates and their smoothing to rid them of the irregularities resulting from the randomness in the 
exposure and the number of deaths in order to facilitate dealing with these rates to complete the process of establishing death tables.

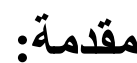

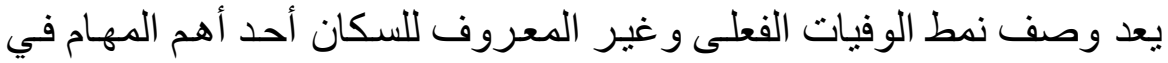

العلوم الاكتوارية. ومن أجل تحقيق ذللك يحسب الخبير الاكتو اري من البيات البيانات الأولية البية

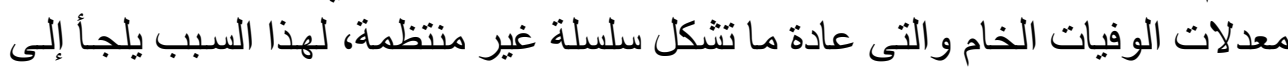

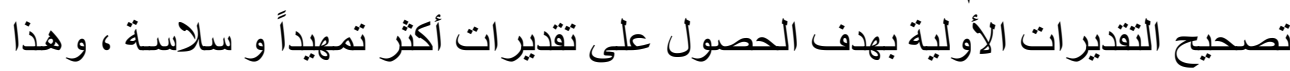

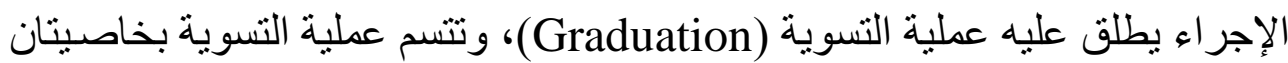

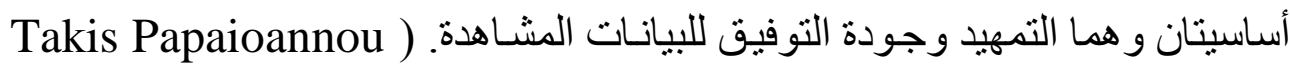

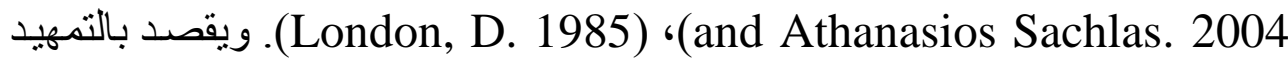

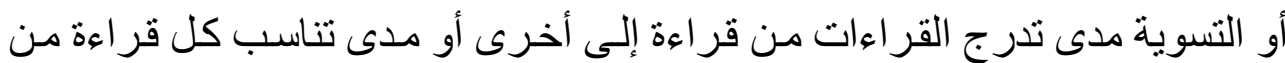

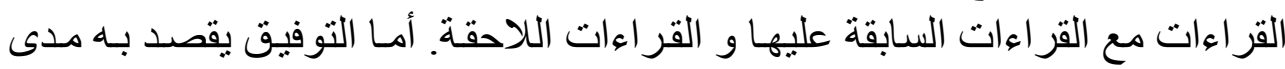

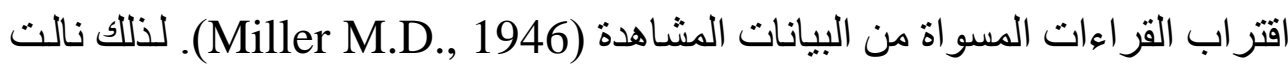

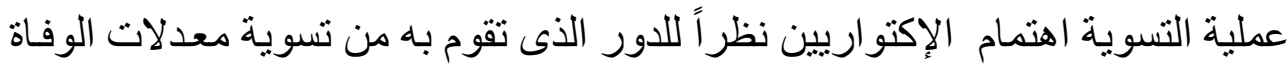

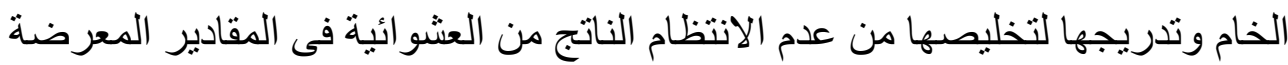

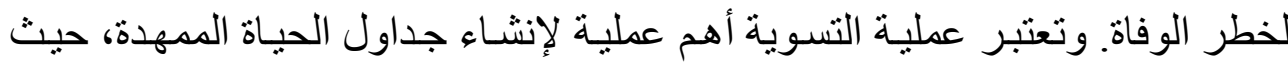

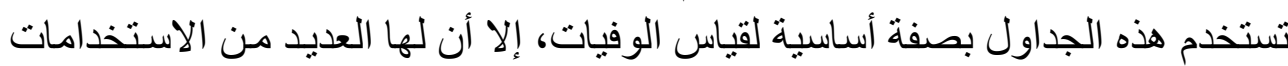

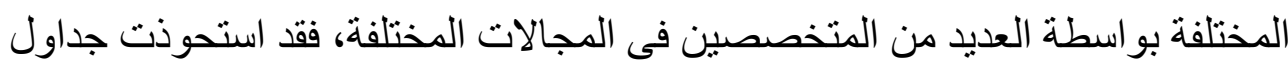

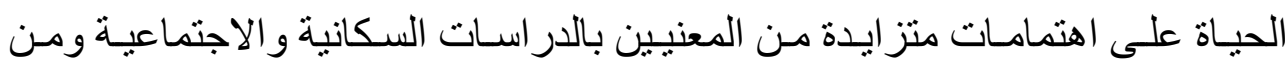

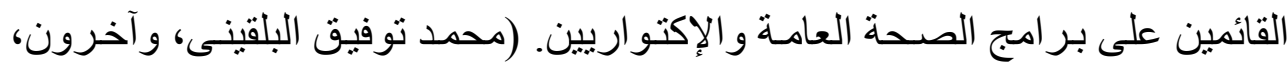
$(r \cdot 14$

وتتقسم طرق تسوية معدلات الوفاة الخام إلى نو عين من الطرق: النوع الأول: يتمثل

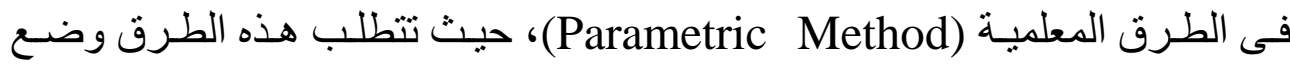

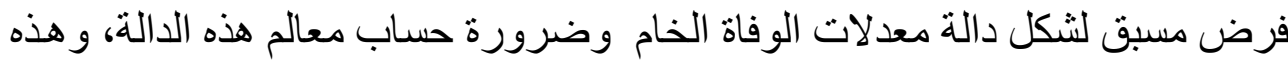

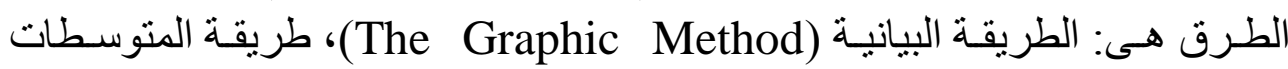
المعدلة (Adjusted Average Method)، طريقة الجداول النمطية (Standard) 
(The Graduation by Interpolation) طريقة التسوية بالتوليد البينى (Tables)

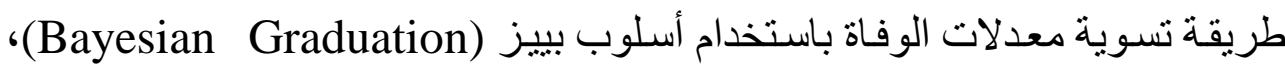

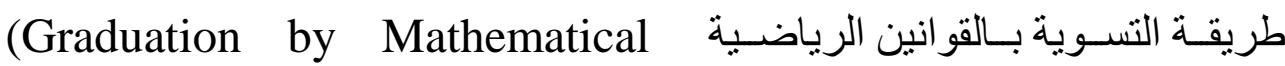
Formula)

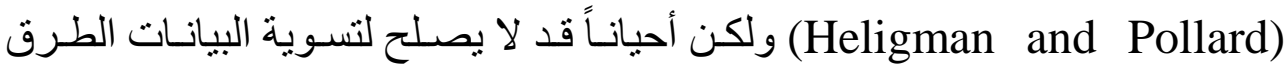

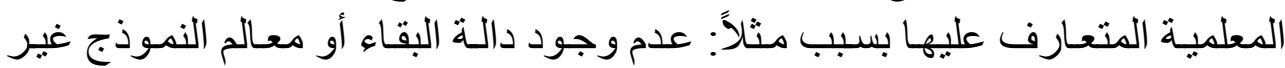

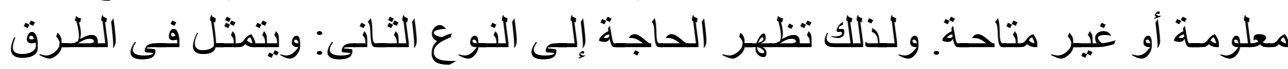

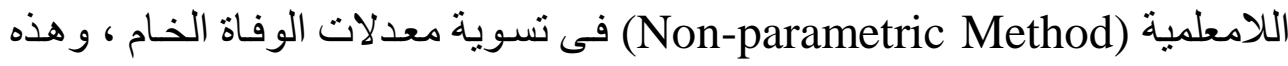

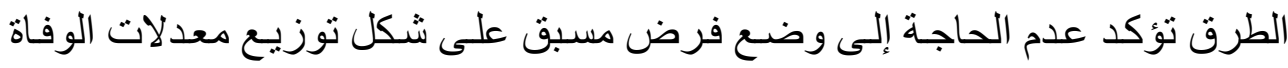

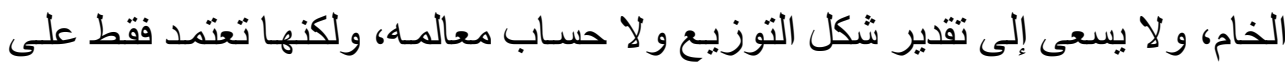

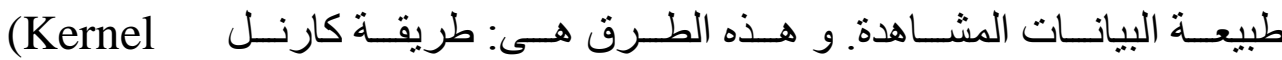
(Moving Weighted Average) (Smoothing)

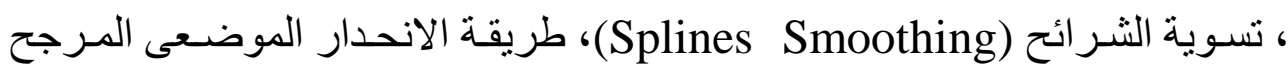
(Generalised (Locally-weighted Regression) (Debon A, .(Whittaker - Henderson ) وطريقة (Additive Models)

.Montes F, and Sala R., 2006)

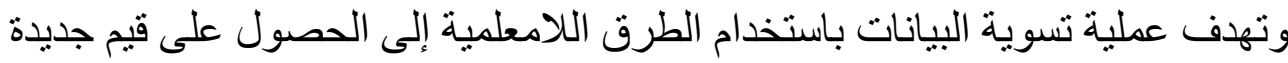

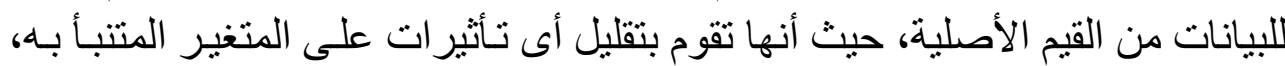

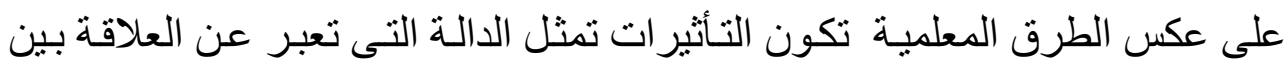

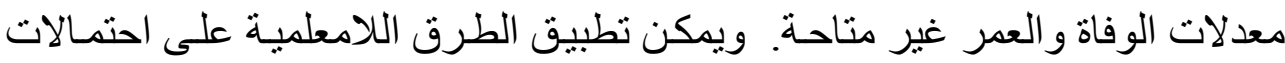

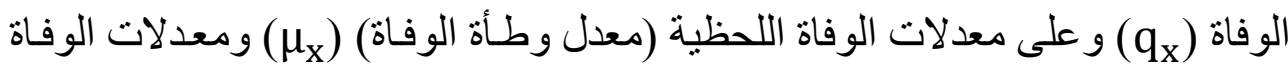

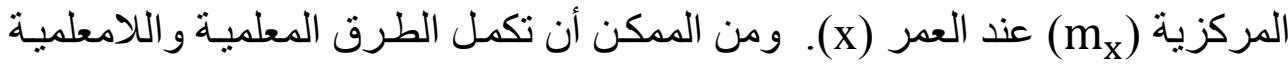

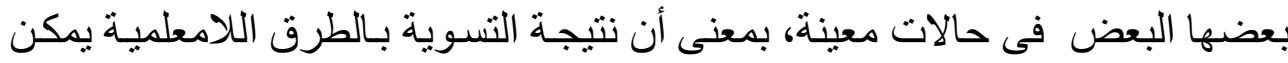

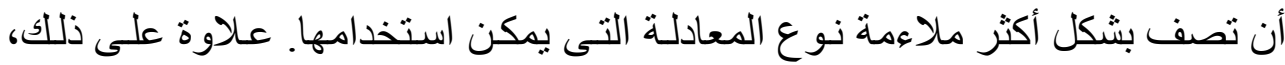

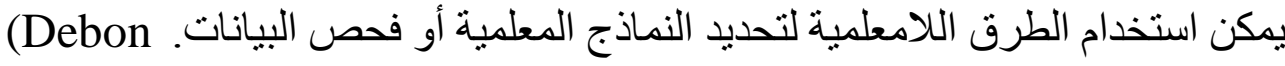

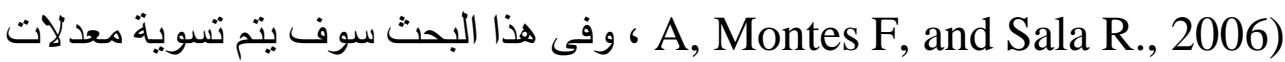




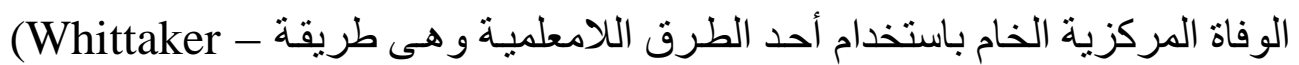
Henderson)

وتتمثل مشكلة البحث فى أننا نجد فى الدول التى تتو افر فيها تعدادات سكانية دقيقة

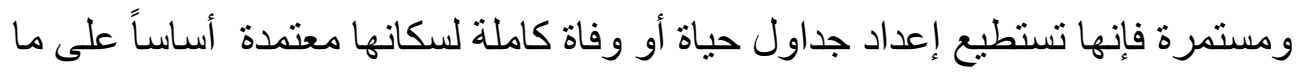

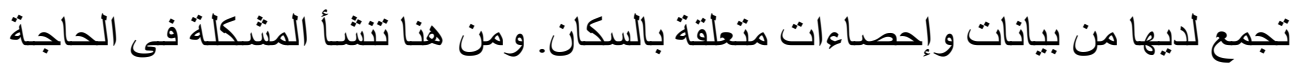

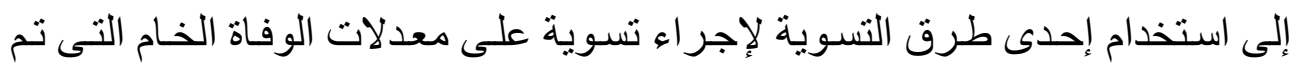
التوصل إليها من التعدادات للحصول على معدلات التهولة الوفاة الممهذة أو المسواة.

وبالتالى يهدف هذا البحث إلى استخدام طريقة Whittaker - Henderson)

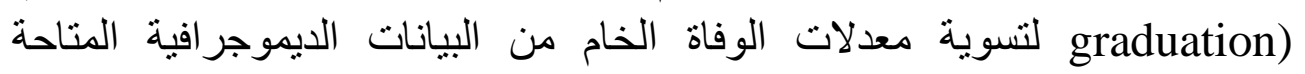

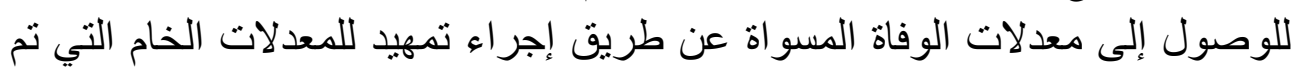

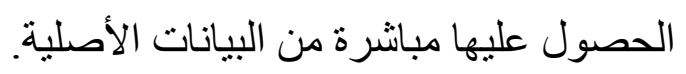

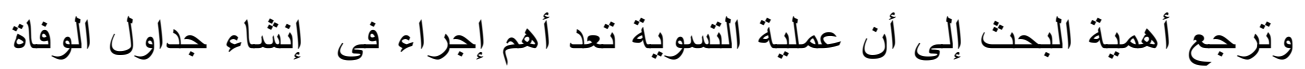

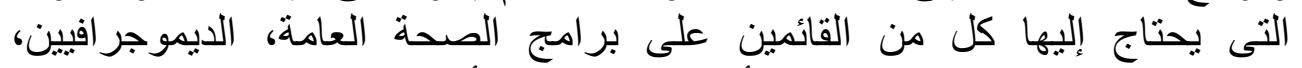
الإكتو اريين، و القائمين على تقدير الأقساط بشركات التأمين على الحياة.

\section{وقد تناولت الدراسات السابقة الآتى:}

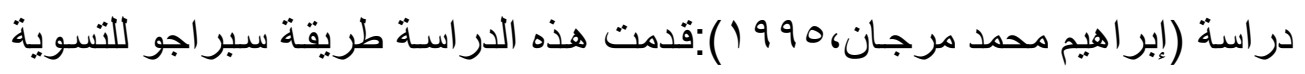

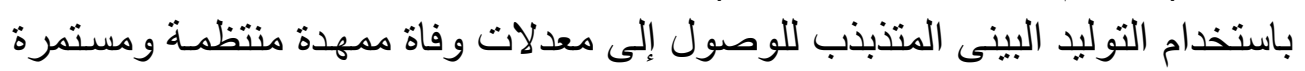

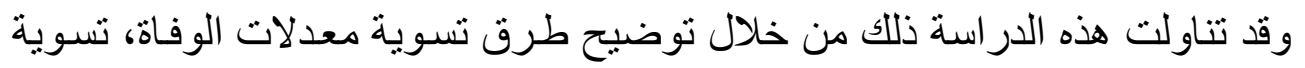
سبر اجو بالتوليد البينى المتذبذب وتطبيقها على بيانات فعلية مصرية.

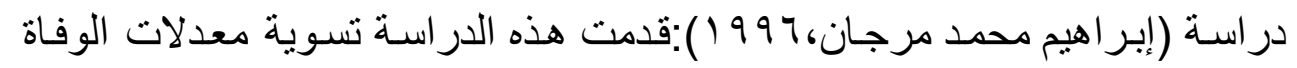

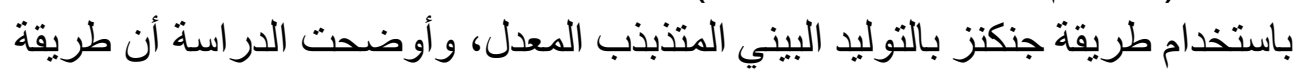

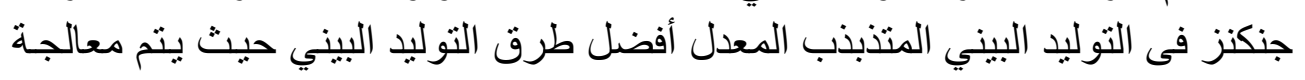

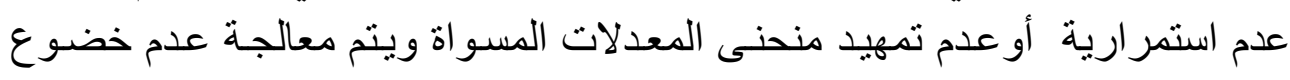

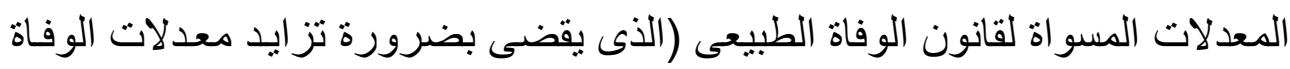


مع نز ايد الأعمار فيما عدا السنوات القليلة الأولى من العمر) بالإضـافة إلى تحقيق

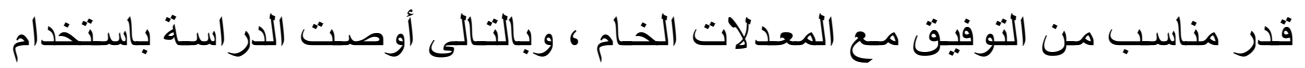

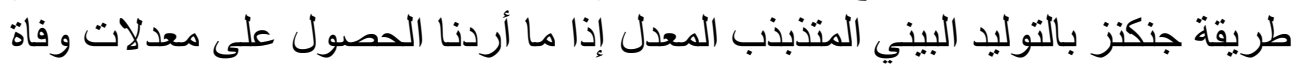

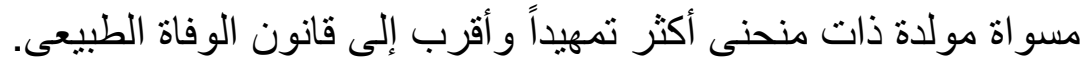

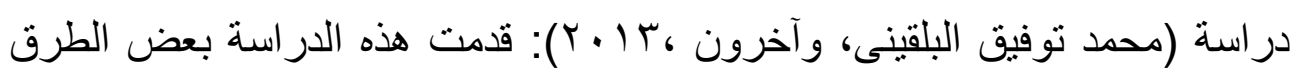

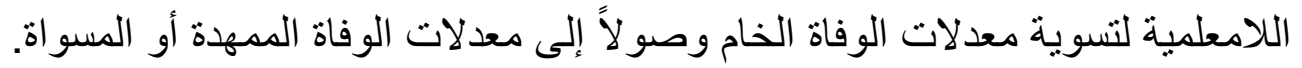

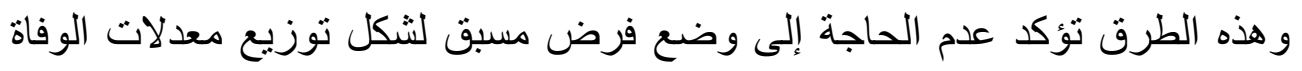

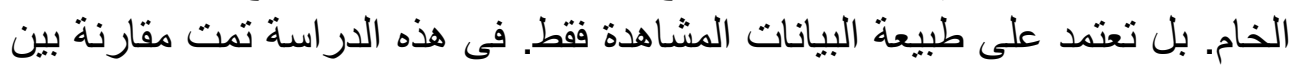

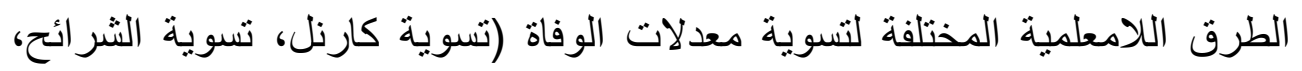

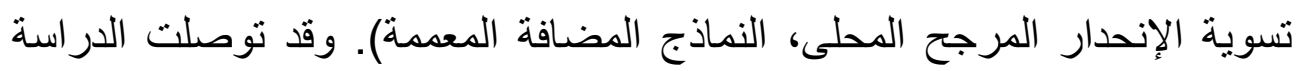

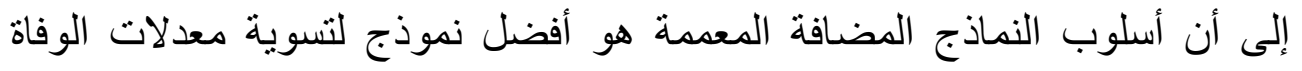
الخام للوصول إلى معدلات الوفاة الممهدة للإعتماد عليها عند إنشاء جدول العيدة الحياة.

دراسة (Siu-Hang Li and Wai-Sum Chan, 2004): قدمت هذه الدراسة تسوية معدلات الوفاة الخام بإستخدام طريقة (Whittaker - Henderson).

دراسة (Marielynn E. Chanco. 2016): هدفت هذه الدراسة إلى إيجاد تقدير

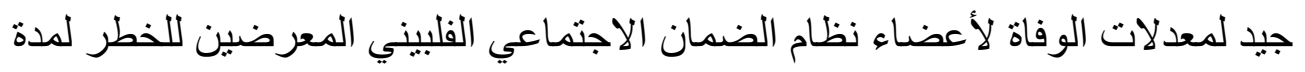

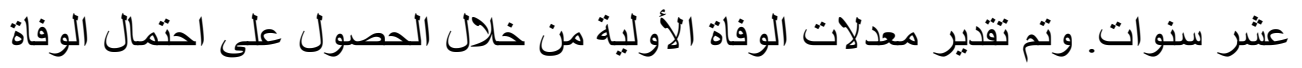

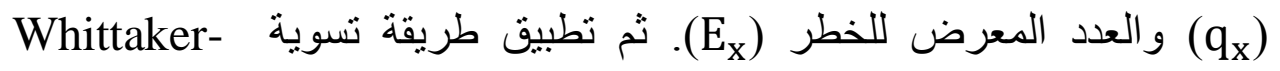
Henderson البيانات الأصلية.

طريقة (Whittaker - Henderson graduation):

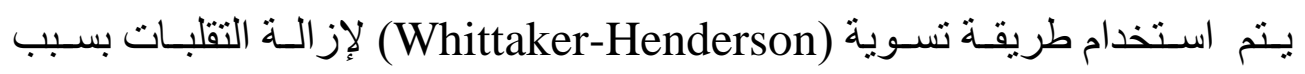

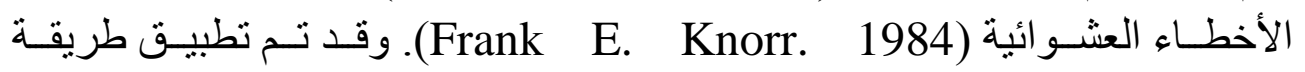
Marielynn E. Chanco. ) على احتمالات الوفاة (Whittaker-Henderson) 


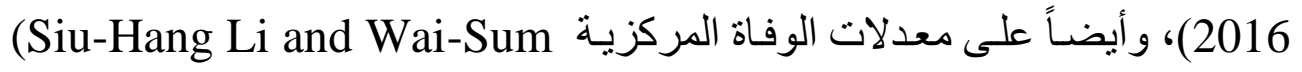

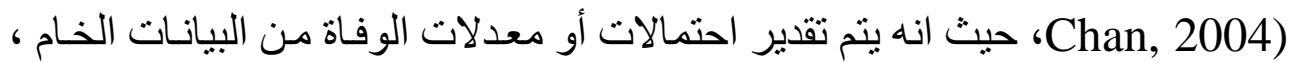

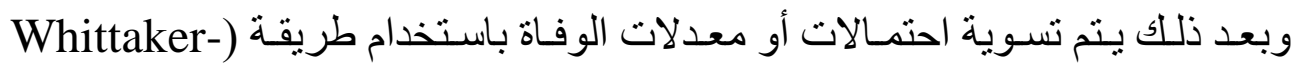
(Henderson

$$
\begin{gathered}
M=F+\lambda S=\sum_{x=0}^{n} W_{x}\left(m_{x}^{g}-m_{x}\right)^{2}+\lambda \sum_{x=0}^{n-d}\left(\Delta^{d} m_{x}^{g}\right)^{2} \\
F=\sum_{x=0}^{n} W_{x}\left(m_{x}^{g}-m_{x}\right)^{2} \Rightarrow \text { measure of fitness. } \\
S=\sum_{x=0}^{n-z}\left(\Delta^{z} m_{x}^{g}\right)^{2} \Rightarrow \text { measure of smoothness. } \\
\Delta \Rightarrow \text { forward difference operator. }
\end{gathered}
$$$$
\text { d عدد مرات الفروق التى سوف يتم تطبيقها. }
$$$$
\text { قيم معدلات الوفاة المركزية المسواه عند العمر (x). }
$$$$
\text { (x) : قيم معدلات الوفاة المركزية الخام عند العمر (x). }
$$$$
\text { n : أعلى عمر. }
$$$$
\text { X }
$$

ג : عدد غير سالب يمثل ثابت التسوية (حيث يعكس الأهمية النسبية للتسوية على 
بتصغير (F)، يجعل قيم (F)

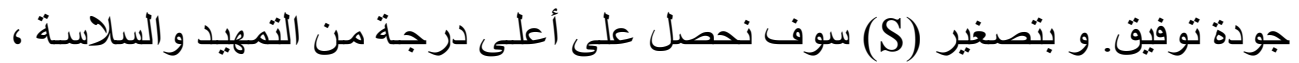

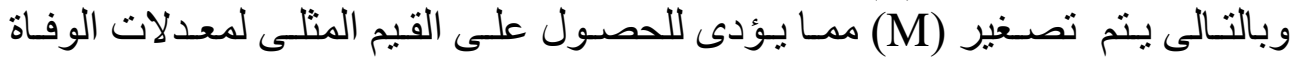

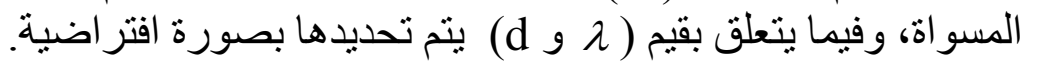

و تعد طريقة (Whittaker - Henderson graduation) (لمقيقة (London, D. 1985) هى الطريقة المثلى التى تقلل من:

(weighted sum of squared deviations) $+\lambda$ (sum of squared zth differences)

لأنها تعد واحدة من أكثر الطرق شيو عاً لتسوية وتوفيق معدلات الوفاة الخام، حيث الطيث

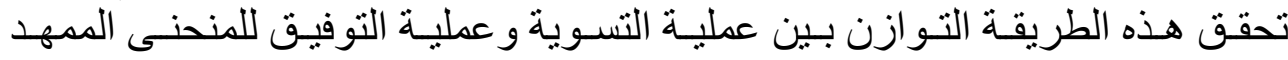

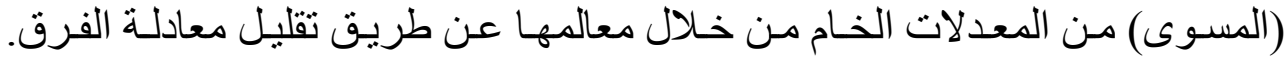

(Marielynn E. Chanco. 2016)

وسوف يتم تطبيق طريقة (Whittaker - Henderson graduation) بإستخدام

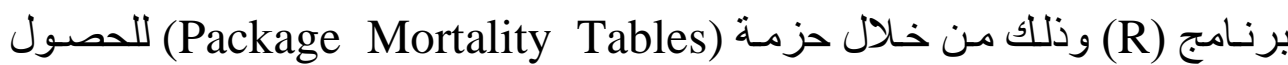

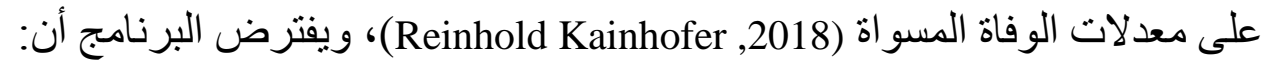

(Smoothing parameter) معلمة التسوية تثشير المعلمة ( $)$ ) إلى أهية التسوية. حيث أن انخفاض قيمة (

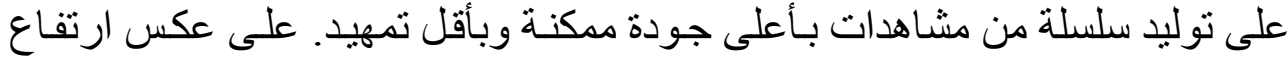

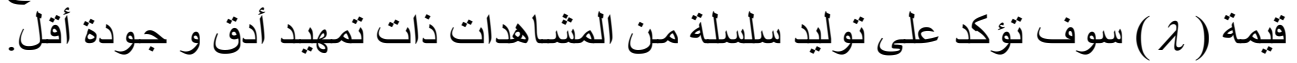

(Walter B. Lowrie., 1982)

(order of differences) درجة الفروق : d 


\section{الاراسة التطبيقية:}

\section{اعتمدت الدراسة التطبيقية على ما يلى:}

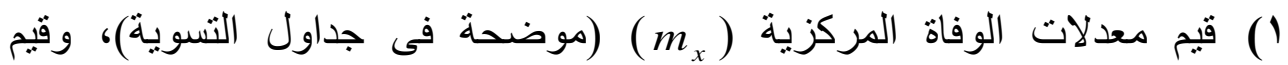

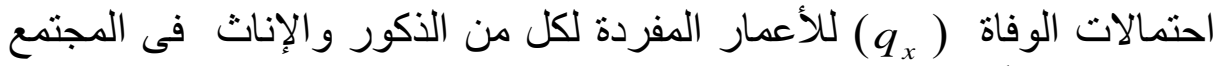

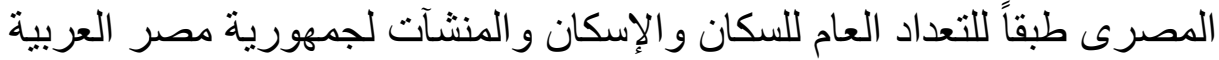

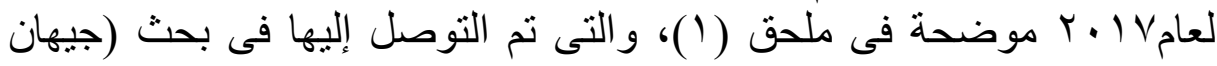

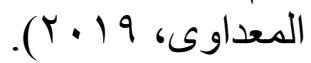

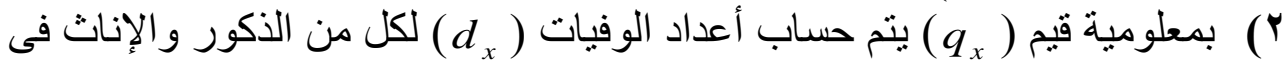

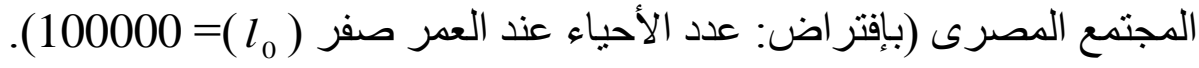
r) يتم حساب العدد المعرض للخطر ( المصرى بإستخدام العلاقة التالية:

$$
m_{x}=\frac{d_{x}}{E_{x}}
$$

(Johnny Siu-Hang Li, Mary R. Hardy, and Ken Seng Tan. 2008)

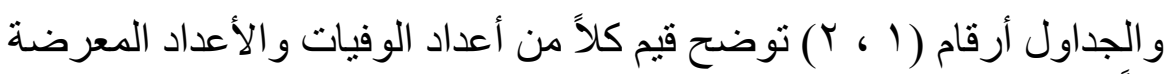

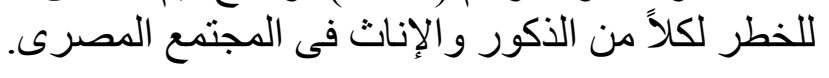


جدول (1): يوضح أعداد الوفيات والأعداد المعرضة للخطر للأكور.

\begin{tabular}{|c|c|c||c|c|c||c|c|c||c|c|c|}
\hline Age & $d_{x}$ & $E_{x}$ & Age & $d_{x}$ & $E_{x}$ & Age & $d_{x}$ & $E_{x}$ & Age & $d_{x}$ & $E_{x}$ \\
\hline 0 & 1552 & 99224 & 29 & 126 & 95999 & 58 & 1501 & 81327 & 87 & 2862 & 9195 \\
\hline 1 & 113 & 98392 & 30 & 132 & 95869 & 59 & 1609 & 79772 & 88 & 2392 & 6568 \\
\hline 2 & 113 & 98279 & 31 & 137 & 95735 & 60 & 1709 & 78113 & 89 & 1854 & 4445 \\
\hline 3 & 100 & 98173 & 32 & 143 & 95595 & 61 & 1802 & 76358 & 90 & 1339 & 2848 \\
\hline 4 & 87 & 98080 & 33 & 150 & 95448 & 62 & 1886 & 74513 & 91 & 903 & 1727 \\
\hline 5 & 74 & 98000 & 34 & 158 & 95294 & 63 & 2051 & 72545 & 92 & 570 & 990 \\
\hline 6 & 61 & 97932 & 35 & 166 & 95132 & 64 & 2200 & 70419 & 93 & 337 & 536 \\
\hline 7 & 49 & 97877 & 36 & 173 & 94962 & 65 & 2331 & 68153 & 94 & 187 & 274 \\
\hline 8 & 49 & 97828 & 37 & 181 & 94785 & 66 & 2444 & 65765 & 95 & 97 & 132 \\
\hline 9 & 49 & 97779 & 38 & 199 & 94595 & 67 & 2539 & 63273 & 96 & 47 & 60 \\
\hline 10 & 50 & 97730 & 39 & 218 & 94387 & 68 & 2659 & 60674 & 97 & 21 & 26 \\
\hline 11 & 50 & 97680 & 40 & 236 & 94160 & 69 & 2755 & 57967 & 98 & 9 & 10 \\
\hline 12 & 51 & 97629 & 41 & 254 & 93915 & 70 & 2827 & 55176 & 99 & 4 & 4 \\
\hline 13 & 58 & 97575 & 42 & 272 & 93652 & 71 & 2874 & 52326 & $100+$ & 2 & 2 \\
\hline 14 & 66 & 97513 & 43 & 308 & 93361 & 72 & 2898 & 49439 & & & \\
\hline 15 & 74 & 97443 & 44 & 344 & 93035 & 73 & 2900 & 46540 & & & \\
\hline 16 & 81 & 97365 & 45 & 380 & 92673 & 74 & 2882 & 43649 & & & \\
\hline 17 & 89 & 97280 & 46 & 415 & 92275 & 75 & 2844 & 40786 & & & \\
\hline 18 & 92 & 97189 & 47 & 449 & 91843 & 76 & 2788 & 37971 & & & \\
\hline 19 & 96 & 97095 & 48 & 540 & 91349 & 77 & 2716 & 35219 & & & \\
\hline 20 & 99 & 96998 & 49 & 629 & 90764 & 78 & 2630 & 32546 & & & \\
\hline 21 & 102 & 96897 & 50 & 717 & 90091 & 79 & 2532 & 29965 & & & \\
\hline 22 & 106 & 96793 & 51 & 802 & 89331 & 80 & 2425 & 27486 & & & \\
\hline 23 & 108 & 96687 & 52 & 885 & 88488 & 81 & 2309 & 25120 & & & \\
\hline 24 & 110 & 96578 & 53 & 993 & 87549 & 82 & 2187 & 22872 & & & \\
\hline 25 & 112 & 96467 & 54 & 1098 & 86504 & 83 & 2060 & 20749 & & & \\
\hline 26 & 114 & 96355 & 55 & 1198 & 85356 & 84 & 2790 & 18323 & & & \\
\hline 27 & 115 & 96240 & 56 & 1294 & 84109 & 85 & 3151 & 15352 & & & \\
\hline 28 & 121 & 96122 & 57 & 1385 & 82770 & 86 & 3151 & 12201 & & & \\
\hline
\end{tabular}


جدول (r): يوضح أعداد الوفيات والأعداد المعرضة للخطر للإناث.

\begin{tabular}{|c|c|c||c|c|c||c|c|c||c|c|c|}
\hline Age & $d_{x}$ & $E_{x}$ & Age & $d_{x}$ & $E_{x}$ & Age & $d_{x}$ & $E_{x}$ & Age & $d_{x}$ & $E_{x}$ \\
\hline 0 & 1393 & 99304 & 29 & 66 & 97160 & 58 & 1086 & 87737 & 87 & 2663 & 8078 \\
\hline 1 & 100 & 98557 & 30 & 71 & 97092 & 59 & 1213 & 86588 & 88 & 2160 & 5666 \\
\hline 2 & 100 & 98458 & 31 & 77 & 97018 & 60 & 1334 & 85314 & 89 & 1632 & 3770 \\
\hline 3 & 87 & 98364 & 32 & 82 & 96938 & 61 & 1449 & 83922 & 90 & 1152 & 2379 \\
\hline 4 & 74 & 98284 & 33 & 88 & 96853 & 62 & 1558 & 82419 & 91 & 762 & 1422 \\
\hline 5 & 62 & 98216 & 34 & 93 & 96763 & 63 & 1821 & 80730 & 92 & 473 & 804 \\
\hline 6 & 49 & 98160 & 35 & 99 & 96666 & 64 & 2065 & 78787 & 93 & 275 & 431 \\
\hline 7 & 36 & 98118 & 36 & 105 & 96564 & 65 & 2288 & 76610 & 94 & 150 & 218 \\
\hline 8 & 35 & 98082 & 37 & 110 & 96457 & 66 & 2488 & 74222 & 95 & 77 & 104 \\
\hline 9 & 34 & 98047 & 38 & 123 & 96340 & 67 & 2664 & 71646 & 96 & 37 & 47 \\
\hline 10 & 33 & 98014 & 39 & 136 & 96210 & 68 & 2931 & 68849 & 97 & 17 & 20 \\
\hline 11 & 31 & 97982 & 40 & 149 & 96068 & 69 & 3156 & 65806 & 98 & 7 & 8 \\
\hline 12 & 30 & 97951 & 41 & 162 & 95912 & 70 & 3337 & 62560 & 99 & 3 & 3 \\
\hline 13 & 33 & 97920 & 42 & 175 & 95743 & 71 & 3474 & 59155 & $100+$ & 2 & 2 \\
\hline 14 & 36 & 97885 & 43 & 197 & 95557 & 72 & 3567 & 55634 & & & \\
\hline 15 & 39 & 97848 & 44 & 219 & 95349 & 73 & 3617 & 52042 & & & \\
\hline 16 & 42 & 97807 & 45 & 240 & 95119 & 74 & 3626 & 48420 & & & \\
\hline 17 & 45 & 97764 & 46 & 262 & 94868 & 75 & 3597 & 44809 & & & \\
\hline 18 & 45 & 97719 & 47 & 283 & 94596 & 76 & 3533 & 41244 & & & \\
\hline 19 & 46 & 97674 & 48 & 336 & 94286 & 77 & 3438 & 37758 & & & \\
\hline 20 & 46 & 97628 & 49 & 389 & 93924 & 78 & 3316 & 34381 & & & \\
\hline 21 & 46 & 97582 & 50 & 442 & 93508 & 79 & 3171 & 31138 & & & \\
\hline 22 & 47 & 97535 & 51 & 493 & 93041 & 80 & 3007 & 28049 & & & \\
\hline 23 & 48 & 97488 & 52 & 544 & 92522 & 81 & 2830 & 25130 & & & \\
\hline 24 & 50 & 97438 & 53 & 630 & 91935 & 82 & 2642 & 22394 & & & \\
\hline 25 & 52 & 97387 & 54 & 714 & 91263 & 83 & 2449 & 19849 & & & \\
\hline 26 & 53 & 97335 & 55 & 796 & 90508 & 84 & 2996 & 17126 & & & \\
\hline 27 & 55 & 97281 & 56 & 876 & 89672 & 85 & 3180 & 14038 & & & \\
\hline 28 & 60 & 97223 & 57 & 954 & 88757 & 86 & 3039 & 10929 & & & \\
\hline
\end{tabular}

ع) تسوية معدلات الوفاة المركزية الخام للأعمار المفردة باستخدام طريقة ويتم إجراء عملية التسوية من (Whittaker - Henderson graduation)

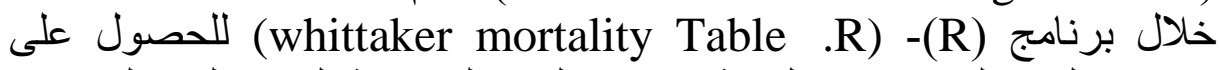

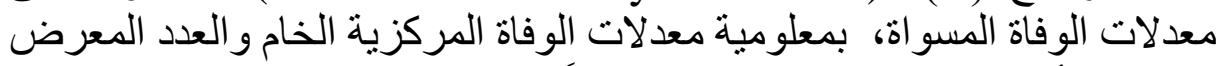

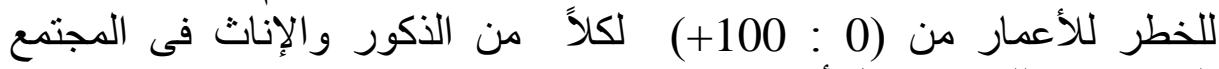

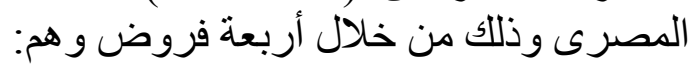

$$
\text { الفرض الأول: (d=2, (d=1) }
$$




$$
\begin{aligned}
& \text { الفرض الثانى: (d=2, (d) } \\
& \text { الفرض الثالث: (d=3 } 1 \text { (d=1) } \\
& \text { الفرض الرابع: (d=3=1/10) } \\
& \text { وموضح ذللك فى الجداول أرقام (س ، ع ): }
\end{aligned}
$$

\begin{tabular}{|c|c|c|c|c|c|}
\hline $\operatorname{age}(x)$ & $m_{x}$ (male) & $\begin{array}{c}m_{x}^{g} \\
\left(\mathbf{d}=\mathbf{2}, \lambda_{=\mathbf{1}}\right)\end{array}$ & $\begin{array}{c}m_{x}^{g} \\
\left(\mathbf{d = 2}, \lambda_{=\mathbf{1} / \mathbf{1 0})}\right.\end{array}$ & $\begin{array}{c}m_{x}^{g} \\
\left(\mathbf{d}=\mathbf{3}, \lambda_{=\mathbf{1}}\right)\end{array}$ & $\begin{array}{c}m_{x}^{g} \\
\left(\mathbf{d = 3}, \lambda_{=\mathbf{1} / \mathbf{1 0})}\right.\end{array}$ \\
\hline 0 & 0.015638754 & 0.005740183 & 0.003371994 & 0.008719792 & 0.006567921 \\
\hline 1 & 0.001144879 & 0.002829522 & 0.002376304 & 0.002718617 & 0.002990081 \\
\hline 2 & 0.001144879 & 0.001609803 & 0.001711787 & 0.001293493 & 0.001618705 \\
\hline 3 & 0.001015061 & 0.001073077 & 0.001275156 & 0.000863891 & 0.001029182 \\
\hline 4 & 0.000885242 & 0.000810663 & 0.000988101 & 0.000712577 & 0.000750585 \\
\hline 5 & 0.000755424 & 0.000666096 & 0.000798581 & 0.000646356 & 0.000610133 \\
\hline 6 & 0.000625606 & 0.000578444 & 0.000673902 & 0.000600824 & 0.000537812 \\
\hline 7 & 0.000495787 & 0.000525143 & 0.000593984 & 0.000559838 & 0.000502734 \\
\hline 8 & 0.000500218 & 0.000498488 & 0.000546433 & 0.000526685 & 0.000490393 \\
\hline 9 & 0.00050465 & 0.000490837 & 0.000522949 & 0.000506159 & 0.000493636 \\
\hline 10 & 0.000509081 & 0.000497602 & 0.000518294 & 0.000501126 & 0.000509123 \\
\hline 11 & 0.000513512 & 0.000517541 & 0.000529303 & 0.000513601 & 0.000535646 \\
\hline 12 & 0.000517943 & 0.000552048 & 0.000554053 & 0.000545554 & 0.000573017 \\
\hline 13 & 0.000597672 & 0.000603048 & 0.00059107 & 0.000597795 & 0.00062103 \\
\hline 14 & 0.0006774 & 0.000667645 & 0.000638376 & 0.000667182 & 0.000678482 \\
\hline 15 & 0.000757129 & 0.000740433 & 0.000693492 & 0.000746644 & 0.00074279 \\
\hline 16 & 0.000836858 & 0.000814678 & 0.000753485 & 0.000826652 & 0.000810107 \\
\hline 17 & 0.000916587 & 0.000883529 & 0.000815174 & 0.00089805 & 0.000875948 \\
\hline 18 & 0.000951287 & 0.000941894 & 0.000875558 & 0.000955436 & 0.0009362 \\
\hline 19 & 0.000985987 & 0.000989419 & 0.000932411 & 0.000999107 & 0.00098814 \\
\hline 20 & 0.001020687 & 0.001028035 & 0.000984358 & 0.001032794 & 0.001030831 \\
\hline 21 & 0.001055387 & 0.001060054 & 0.001030851 & 0.001060503 & 0.001064978 \\
\hline
\end{tabular}

جدول (r): معدلات الوفاة المركزية المسواة للاكور 
تابع جدول (َّ):

\begin{tabular}{|c|c|c|c|c|c|}
\hline $\mathbf{a g e}(\mathbf{x})$ & $m_{x}$ (male) & $\begin{array}{c}m_{x}^{g} \\
(\mathbf{d}=\mathbf{2}, \lambda=\mathbf{1})\end{array}$ & $\begin{array}{c}m_{x}^{g} \\
\left(\mathbf{d}=\mathbf{2}, \lambda_{=1 / 10)}\right.\end{array}$ & $\begin{array}{c}m_{x}^{g} \\
\left(\mathbf{d}=\mathbf{3}, \lambda_{=1}\right)\end{array}$ & $\begin{array}{c}m_{x}^{g} \\
(\mathbf{d}=\mathbf{3}, \boldsymbol{\lambda}=\mathbf{1} \mathbf{1 0} \mathbf{)}\end{array}$ \\
\hline $\mathbf{2 2}$ & 0.001090086 & 0.001087295 & 0.001072086 & 0.001084846 & 0.001092477 \\
\hline $\mathbf{2 3}$ & 0.001112054 & 0.00111123 & 0.001108899 & 0.001107254 & 0.001115906 \\
\hline $\mathbf{2 4}$ & 0.001134022 & 0.001133948 & 0.00114267 & 0.001129172 & 0.001138106 \\
\hline $\mathbf{2 5}$ & 0.00115599 & 0.001157852 & 0.001175089 & 0.001152686 & 0.001161809 \\
\hline $\mathbf{2 6}$ & 0.001177958 & 0.001185572 & 0.001207958 & 0.001180557 & 0.00118936 \\
\hline $\mathbf{2 7}$ & 0.001199926 & 0.001219727 & 0.001243007 & 0.001215586 & 0.001222523 \\
\hline $\mathbf{2 8}$ & 0.001258607 & 0.001262162 & 0.001281725 & 0.001259421 & 0.00126234 \\
\hline $\mathbf{2 9}$ & 0.001317288 & 0.001312067 & 0.001325139 & 0.001311476 & 0.001309106 \\
\hline $\mathbf{3 0}$ & 0.001375969 & 0.001368 & 0.001374073 & 0.001369923 & 0.001362658 \\
\hline $\mathbf{3 1}$ & 0.00143465 & 0.001429044 & 0.001429357 & 0.001433022 & 0.001422749 \\
\hline $\mathbf{3 2}$ & 0.001493331 & 0.001495282 & 0.001491979 & 0.001499967 & 0.001489404 \\
\hline $\mathbf{3 3}$ & 0.001576123 & 0.001567622 & 0.001563175 & 0.001571042 & 0.001563173 \\
\hline $\mathbf{3 4}$ & 0.001658915 & 0.001646819 & 0.001644414 & 0.001647561 & 0.001645285 \\
\hline $\mathbf{3 5}$ & 0.001741708 & 0.001735021 & 0.001737639 & 0.001732821 & 0.001737786 \\
\hline $\mathbf{3 6}$ & 0.0018245 & 0.001836636 & 0.001845403 & 0.001832567 & 0.001843523 \\
\hline $\mathbf{3 7}$ & 0.001907292 & 0.001958101 & 0.00197087 & 0.001953852 & 0.0019659 \\
\hline $\mathbf{3 8}$ & 0.002107121 & 0.002105623 & 0.002117602 & 0.002102068 & 0.002108455 \\
\hline $\mathbf{3 9}$ & 0.00230695 & 0.002278973 & 0.002288969 & 0.002277819 & 0.002274545 \\
\hline $\mathbf{4 0}$ & 0.002506779 & 0.002477606 & 0.002488889 & 0.002480054 & 0.002467841 \\
\hline $\mathbf{4 1}$ & 0.002706608 & 0.002704599 & 0.002722372 & 0.002710214 & 0.002693082 \\
\hline $\mathbf{4 2}$ & 0.002906437 & 0.002968136 & 0.002995813 & 0.002973889 & 0.002956665 \\
\hline $\mathbf{4 3}$ & 0.003303812 & 0.003279078 & 0.003316821 & 0.003278595 & 0.003266831 \\
\hline $\mathbf{4 4}$ & 0.003701188 & 0.00364126 & 0.003693232 & 0.003630407 & 0.003633586 \\
\hline $\mathbf{4 5}$ & 0.004098564 & 0.004062257 & 0.004134113 & 0.004040643 & 0.004069305 \\
\hline $\mathbf{4 6}$ & 0.00449594 & 0.004560728 & 0.004650249 & 0.004532836 & 0.004588878 \\
\hline $\mathbf{4 7}$ & 0.004893316 & 0.005167772 & 0.00525369 & 0.005141287 & 0.00520834 \\
\hline $\mathbf{4 8}$ & 0.005913929 & 0.005915611 & 0.005955646 & 0.005895721 & 0.005941387 \\
\hline $\mathbf{4 9}$ & 0.006934542 & 0.006798442 & 0.006761488 & 0.006796792 & 0.006794673 \\
\hline & & & & & \\
\hline
\end{tabular}

12 


\begin{tabular}{|c|c|c|c|c|c|}
\hline $\operatorname{age}(x)$ & $m_{x}$ (male) & $\begin{array}{c}m_{x}^{g} \\
\left(\mathbf{d}=2, \lambda_{=1}\right)\end{array}$ & $\begin{array}{c}m_{x}^{g} \\
\left(\mathbf{d = 2}, \lambda_{=\mathbf{1} / \mathbf{1 0})}\right.\end{array}$ & $\begin{array}{c}m_{x}^{g} \\
\left(\mathbf{d}=3, \lambda_{=1}\right)\end{array}$ & $\begin{array}{c}m_{x}^{g} \\
\left(\mathrm{~d}=\mathbf{3}, \lambda_{=\mathbf{1} / 10)}\right.\end{array}$ \\
\hline 50 & 0.007955156 & 0.007794758 & 0.007672509 & 0.007820708 & 0.007766528 \\
\hline 51 & 0.008975769 & 0.008883335 & 0.008687415 & 0.00893659 & 0.008848001 \\
\hline 52 & 0.009996382 & 0.010052527 & 0.009803523 & 0.010122448 & 0.010025248 \\
\hline 53 & 0.011344099 & 0.011298609 & 0.011017301 & 0.011368052 & 0.011282232 \\
\hline 54 & 0.012691816 & 0.012607807 & 0.012323691 & 0.012665461 & 0.012603361 \\
\hline 55 & 0.014039534 & 0.013968625 & 0.01371861 & 0.014009908 & 0.013977407 \\
\hline 56 & 0.015387251 & 0.01538019 & 0.01520106 & 0.015404063 & 0.015401112 \\
\hline 57 & 0.016734968 & 0.016854995 & 0.016774376 & 0.016859333 & 0.016881181 \\
\hline 58 & 0.01845089 & 0.018413811 & 0.01844616 & 0.018391693 & 0.018434182 \\
\hline 59 & 0.020166812 & 0.020068994 & 0.020226415 & 0.020016963 & 0.020084968 \\
\hline 60 & 0.021882734 & 0.021842164 & 0.022128536 & 0.021762895 & 0.02186566 \\
\hline 61 & 0.023598656 & 0.023774858 & 0.024169076 & 0.023679255 & 0.02381357 \\
\hline 62 & 0.025314578 & 0.025926704 & 0.026365692 & 0.025830392 & 0.025966758 \\
\hline 63 & 0.02827844 & 0.028351952 & 0.028732537 & 0.028264887 & 0.028356975 \\
\hline 64 & 0.031242302 & 0.031039112 & 0.031272211 & 0.030978438 & 0.031002101 \\
\hline 65 & 0.034206164 & 0.033954138 & 0.033979324 & 0.033930553 & 0.033904576 \\
\hline 66 & 0.037170027 & 0.037066998 & 0.036843423 & 0.037076834 & 0.037052039 \\
\hline 67 & 0.040133889 & 0.040361114 & 0.03985109 & 0.040390307 & 0.040417528 \\
\hline 68 & 0.043831892 & 0.043823187 & 0.042987056 & 0.043858806 & 0.043958231 \\
\hline 69 & 0.047529895 & 0.047410031 & 0.046234139 & 0.04746556 & 0.047614169 \\
\hline 70 & 0.051227898 & 0.051065771 & 0.049579213 & 0.051187213 & 0.051311429 \\
\hline 71 & 0.054925901 & 0.054732151 & 0.053019159 & 0.054994195 & 0.054969878 \\
\hline 72 & 0.058623904 & 0.058354881 & 0.056566585 & 0.058845057 & 0.058515488 \\
\hline 73 & 0.062321907 & 0.061889757 & 0.060255178 & 0.062674956 & 0.061897002 \\
\hline 74 & 0.066019909 & 0.065312239 & 0.064144584 & 0.066387243 & 0.065105645 \\
\hline 75 & 0.069717912 & 0.068633256 & 0.068324906 & 0.069863628 & 0.068195462 \\
\hline 76 & 0.073415915 & 0.071922286 & 0.072920983 & 0.073008343 & 0.071301314 \\
\hline 77 & 0.077113918 & 0.075336644 & 0.078096745 & 0.075830642 & 0.074652414 \\
\hline
\end{tabular}


تابع جدول (r):

\begin{tabular}{|c|c|c|c|c|c|}
\hline age(x) & $m_{x}$ (male) & $\begin{array}{c}m_{x}^{g} \\
(\mathbf{d = 2}, \lambda=\mathbf{1})\end{array}$ & $\begin{array}{c}m_{x}^{g} \\
\left(\mathbf{d = 2}, \lambda_{\mathbf{= 1} / \mathbf{1 0})}\right.\end{array}$ & $\begin{array}{c}m_{x}^{g} \\
(\mathbf{d = 3}, \boldsymbol{\lambda}=\mathbf{1})\end{array}$ & $\begin{array}{c}m_{x}^{g} \\
(\mathbf{d = 3}, \boldsymbol{\lambda}=\mathbf{1 / 1 0})\end{array}$ \\
\hline $\mathbf{7 8}$ & 0.080811921 & 0.079154705 & 0.084059906 & 0.078549207 & 0.078581528 \\
\hline $\mathbf{7 9}$ & 0.084509924 & 0.08381136 & 0.091067016 & 0.081685326 & 0.08353265 \\
\hline $\mathbf{8 0}$ & 0.088207927 & 0.089936295 & 0.09942828 & 0.086118516 & 0.090071476 \\
\hline $\mathbf{8 1}$ & 0.09190593 & 0.098396367 & 0.109510438 & 0.093108375 & 0.098901766 \\
\hline $\mathbf{8 2}$ & 0.095603933 & 0.110333434 & 0.121734019 & 0.104302876 & 0.110886076 \\
\hline $\mathbf{8 3}$ & 0.099301936 & 0.127150446 & 0.136558163 & 0.121705457 & 0.12706268 \\
\hline $\mathbf{8 4}$ & 0.152284175 & 0.150299576 & 0.154441846 & 0.14745853 & 0.148647317 \\
\hline $\mathbf{8 5}$ & 0.205266414 & 0.180534973 & 0.175764981 & 0.183292828 & 0.177023811 \\
\hline $\mathbf{8 6}$ & 0.258248653 & 0.218380455 & 0.200900981 & 0.230176112 & 0.213794374 \\
\hline $\mathbf{8 7}$ & 0.311230892 & 0.26438347 & 0.230263735 & 0.287969269 & 0.260857887 \\
\hline $\mathbf{8 8}$ & 0.364213131 & 0.319317943 & 0.264341964 & 0.355230015 & 0.32051335 \\
\hline $\mathbf{8 9}$ & 0.41719537 & 0.384344352 & 0.303724999 & 0.429258428 & 0.395591914 \\
\hline $\mathbf{9 0}$ & 0.470177609 & 0.461112127 & 0.349122161 & 0.506340849 & 0.489620214 \\
\hline $\mathbf{9 1}$ & 0.523159849 & 0.551811092 & 0.401378117 & 0.582089256 & 0.607017491 \\
\hline $\mathbf{9 2}$ & 0.576142088 & 0.659197954 & 0.461486919 & 0.651809617 & 0.753331584 \\
\hline $\mathbf{9 3}$ & 0.629124327 & 0.786627023 & 0.530607295 & 0.710888697 & 0.935523426 \\
\hline $\mathbf{9 4}$ & 0.682106566 & 0.938106224 & 0.610081141 & 0.75520376 & 1.162314795 \\
\hline $\mathbf{9 5}$ & 0.735088805 & 1.118389003 & 0.701456439 & 0.781533687 & 1.444618881 \\
\hline $\mathbf{9 6}$ & 0.788071044 & 1.333105034 & 0.806515301 & 0.787916754 & 1.796077773 \\
\hline $\mathbf{9 7}$ & 0.841053283 & 1.588929637 & 0.927307507 & 0.773887221 & 2.23373598 \\
\hline $\mathbf{9 8}$ & 0.894035522 & 1.893791824 & 1.066189931 & 0.740537184 & 2.778885287 \\
\hline $\mathbf{9 9}$ & 0.947017761 & 2.257122779 & 1.225872294 & 0.690383668 & 3.458124375 \\
\hline $\mathbf{1 0 0 +}$ & 1.011584064 & 2.690152532 & 1.409470015 & 0.627060132 & 4.304687481 \\
\hline & & & & & \\
\hline
\end{tabular}




\begin{tabular}{|c|c|c|c|c|c|}
\hline age(x) & $\begin{array}{c}m_{x} \\
\text { (Female) } \\
\end{array}$ & $\begin{array}{c}m_{x}^{g} \\
(\mathbf{d}=\mathbf{2}, \lambda=\mathbf{1})\end{array}$ & $\begin{array}{c}m_{x}^{g} \\
(\mathbf{d}=\mathbf{2}, \lambda=\mathbf{1} / \mathbf{1 0})\end{array}$ & $\begin{array}{c}m_{x}^{g} \\
(\mathbf{d}=\mathbf{3}, \lambda=\mathbf{1})\end{array}$ & $\begin{array}{c}m_{x}^{g} \\
\left(\mathbf{d}=\mathbf{3}, \lambda_{=\mathbf{1} / \mathbf{1 0})}\right.\end{array}$ \\
\hline 0 & 0.014024 & 0.005146864 & 0.003109398 & 0.00773815 & 0.005852257 \\
\hline 1 & 0.001013 & 0.00252298 & 0.002142056 & 0.002424962 & 0.002669056 \\
\hline 2 & 0.001013 & 0.001421276 & 0.001506822 & 0.001147019 & 0.001433954 \\
\hline 3 & 0.000884 & 0.00093212 & 0.001093829 & 0.000754077 & 0.000896583 \\
\hline 4 & 0.000756 & 0.000687884 & 0.000823535 & 0.000606982 & 0.000637589 \\
\hline 5 & 0.000627 & 0.000547769 & 0.000644394 & 0.000532977 & 0.000501563 \\
\hline 6 & 0.000499 & 0.000457564 & 0.000524528 & 0.00047616 & 0.000425169 \\
\hline 7 & 0.00037 & 0.000397329 & 0.000444438 & 0.0004241 & 0.000380406 \\
\hline 8 & 0.000358 & 0.000359789 & 0.000391985 & 0.0003802 & 0.000354088 \\
\hline 9 & 0.000346 & 0.000337485 & 0.00035896 & 0.000347698 & 0.000339616 \\
\hline 10 & 0.000333 & 0.000325775 & 0.000340043 & 0.000327573 & 0.00033371 \\
\hline 11 & 0.000321 & 0.00032311 & 0.000331876 & 0.000319956 & 0.000334848 \\
\hline 12 & 0.000309 & 0.000329331 & 0.000332228 & 0.000324676 & 0.000342283 \\
\hline 13 & 0.000338 & 0.000344561 & 0.000339441 & 0.000340906 & 0.000355346 \\
\hline 14 & 0.000368 & 0.000366574 & 0.000351881 & 0.000365968 & 0.000372925 \\
\hline 15 & 0.000398 & 0.000392134 & 0.00036794 & 0.000395519 & 0.000393346 \\
\hline 16 & 0.000427 & 0.000417594 & 0.000386058 & 0.000424333 & 0.00041452 \\
\hline 17 & 0.000457 & 0.00043951 & 0.000404822 & 0.00044774 & 0.000434341 \\
\hline 18 & 0.000462 & 0.000455685 & 0.000423147 & 0.000463378 & 0.000451219 \\
\hline 19 & 0.000466 & 0.000466802 & 0.000440528 & 0.000472173 & 0.000464537 \\
\hline 20 & 0.000471 & 0.000474483 & 0.000456924 & 0.000476908 & 0.000474709 \\
\hline 21 & 0.000476 & 0.000481042 & 0.000472738 & 0.000480762 & 0.000482978 \\
\hline 22 & 0.00048 & 0.000488325 & 0.00048864 & 0.000486146 & 0.000491046 \\
\hline 23 & 0.000497 & 0.000498153 & 0.000505503 & 0.000494624 & 0.000500744 \\
\hline 24 & 0.000514 & 0.000511315 & 0.000524197 & 0.000507 & 0.000513776 \\
\hline 25 & 0.00053 & 0.000528993 & 0.000545642 & 0.000524264 & 0.000531629 \\
\hline
\end{tabular}


تابع جدول (؛ ):

\begin{tabular}{|c|c|c|c|c|c|}
\hline age(x) & $\begin{array}{c}m_{x} \\
\text { (Female) }\end{array}$ & $\begin{array}{c}m_{x}^{g} \\
(\mathbf{d}=\mathbf{2}, \lambda=\mathbf{1})\end{array}$ & $\begin{array}{c}m_{x}^{g} \\
(\mathbf{d}=\mathbf{2}, \lambda=\mathbf{\lambda} / \mathbf{1 0})\end{array}$ & $\begin{array}{c}m_{x}^{g} \\
(\mathbf{d}=\mathbf{3}, \lambda=\mathbf{1})\end{array}$ & $\begin{array}{c}m_{x}^{g} \\
(\mathbf{d}=\mathbf{3}, \boldsymbol{\lambda}=\mathbf{1} / \mathbf{1 0})\end{array}$ \\
\hline $\mathbf{2 6}$ & 0.000547 & 0.000552406 & 0.000570696 & 0.000547897 & 0.000555504 \\
\hline $\mathbf{2 7}$ & 0.000564 & 0.000583228 & 0.000600145 & 0.000579817 & 0.000586231 \\
\hline $\mathbf{2 8}$ & 0.00062 & 0.000623241 & 0.000634621 & 0.000621423 & 0.000624121 \\
\hline $\mathbf{2 9}$ & 0.000677 & 0.000671084 & 0.000674255 & 0.000671786 & 0.000668774 \\
\hline $\mathbf{3 0}$ & 0.000734 & 0.00072437 & 0.000718937 & 0.000728215 & 0.000719217 \\
\hline $\mathbf{3 1}$ & 0.000791 & 0.000781167 & 0.000768547 & 0.000787504 & 0.000774227 \\
\hline $\mathbf{3 2}$ & 0.000848 & 0.000839694 & 0.000823019 & 0.00084676 & 0.000832705 \\
\hline $\mathbf{3 3}$ & 0.000907 & 0.000898975 & 0.000882505 & 0.000904464 & 0.000894106 \\
\hline $\mathbf{3 4}$ & 0.000966 & 0.000959392 & 0.000947524 & 0.000961419 & 0.000958782 \\
\hline $\mathbf{3 5}$ & 0.001025 & 0.001023191 & 0.001019079 & 0.001021227 & 0.001028127 \\
\hline $\mathbf{3 6}$ & 0.001084 & 0.001094874 & 0.001098717 & 0.001089856 & 0.001104425 \\
\hline $\mathbf{3 7}$ & 0.001142 & 0.0011797 & 0.001188353 & 0.001173667 & 0.001190393 \\
\hline $\mathbf{3 8}$ & 0.00128 & 0.001282121 & 0.001290053 & 0.001276881 & 0.001288633 \\
\hline $\mathbf{3 9}$ & 0.001418 & 0.001401469 & 0.001405562 & 0.001399243 & 0.001401189 \\
\hline $\mathbf{4 0}$ & 0.001555 & 0.001535975 & 0.001536753 & 0.001538112 & 0.001529774 \\
\hline $\mathbf{4 1}$ & 0.001693 & 0.001685577 & 0.001686015 & 0.001691675 & 0.001676281 \\
\hline $\mathbf{4 2}$ & 0.00183 & 0.001853523 & 0.001856527 & 0.001860919 & 0.00184331 \\
\hline $\mathbf{4 3}$ & 0.002062 & 0.002044594 & 0.002052183 & 0.002048837 & 0.002034476 \\
\hline $\mathbf{4 4}$ & 0.002294 & 0.002261038 & 0.002277273 & 0.002259009 & 0.002254605 \\
\hline $\mathbf{4 5}$ & 0.002526 & 0.002507653 & 0.002537053 & 0.00249862 & 0.002510208 \\
\hline $\mathbf{4 6}$ & 0.002758 & 0.002795055 & 0.002838067 & 0.002781414 & 0.002809681 \\
\hline $\mathbf{4 7}$ & 0.00299 & 0.003141177 & 0.003188173 & 0.003126796 & 0.0031628 \\
\hline $\mathbf{4 8}$ & 0.003568 & 0.003565031 & 0.003595622 & 0.003551756 & 0.003579176 \\
\hline $\mathbf{4 9}$ & 0.004145 & 0.004065938 & 0.004066656 & 0.004059011 & 0.004066213 \\
\hline $\mathbf{5 0}$ & 0.004723 & 0.004637908 & 0.004606907 & 0.004642303 & 0.004628982 \\
\hline $\mathbf{5 1}$ & 0.0053 & 0.005280214 & 0.005222555 & 0.005297439 & 0.005270993 \\
\hline $\mathbf{5 2}$ & 0.005878 & 0.006001346 & 0.005920918 & 0.006028653 & 0.005994958 \\
\hline & & & & & 8 \\
\hline
\end{tabular}

تابع جدول (\&): 


\begin{tabular}{|c|c|c|c|c|c|}
\hline age(x) & $\begin{array}{c}m_{x} \\
\text { (Female) }\end{array}$ & $\begin{array}{c}m_{x}^{g} \\
(\mathbf{d}=\mathbf{2}, \lambda=\mathbf{1})\end{array}$ & $\begin{array}{c}m_{x}^{g} \\
(\mathbf{d}=\mathbf{2}, \lambda=\mathbf{1 / 1 0})\end{array}$ & $\begin{array}{c}m_{x}^{g} \\
(\mathbf{d}=\mathbf{3}, \lambda=\mathbf{1})\end{array}$ & $\begin{array}{c}m_{x}^{g} \\
(\mathbf{d}=\mathbf{3}, \boldsymbol{\lambda}=\mathbf{1 / 1 0})\end{array}$ \\
\hline $\mathbf{5 3}$ & 0.006851 & 0.006814349 & 0.006710287 & 0.006844079 & 0.006803068 \\
\hline $\mathbf{5 4}$ & 0.007825 & 0.007715116 & 0.007598261 & 0.007743801 & 0.007697135 \\
\hline $\mathbf{5 5}$ & 0.008799 & 0.008698773 & 0.008594056 & 0.008725663 & 0.008681025 \\
\hline $\mathbf{5 6}$ & 0.009772 & 0.009773378 & 0.00971067 & 0.00979689 & 0.009763915 \\
\hline $\mathbf{5 7}$ & 0.010746 & 0.010965077 & 0.010966135 & 0.010978767 & 0.010962714 \\
\hline $\mathbf{5 8}$ & 0.012377 & 0.012309755 & 0.012383142 & 0.012298667 & 0.012302716 \\
\hline $\mathbf{5 9}$ & 0.014007 & 0.013822328 & 0.013985989 & 0.013778195 & 0.013817034 \\
\hline $\mathbf{6 0}$ & 0.015638 & 0.015528636 & 0.015803434 & 0.015452612 & 0.015548171 \\
\hline $\mathbf{6 1}$ & 0.017268 & 0.017488026 & 0.017870043 & 0.01739258 & 0.017548537 \\
\hline $\mathbf{6 2}$ & 0.018899 & 0.01979742 & 0.020224682 & 0.019700967 & 0.01987694 \\
\hline $\mathbf{6 3}$ & 0.022554 & 0.022558114 & 0.022904026 & 0.022469281 & 0.022589392 \\
\hline $\mathbf{6 4}$ & 0.02621 & 0.025767303 & 0.025928016 & 0.02571162 & 0.025726597 \\
\hline $\mathbf{6 5}$ & 0.029866 & 0.029385639 & 0.029304278 & 0.02938496 & 0.029310012 \\
\hline $\mathbf{6 6}$ & 0.033522 & 0.033384418 & 0.033031303 & 0.033441503 & 0.033341369 \\
\hline $\mathbf{6 7}$ & 0.037178 & 0.037765708 & 0.037099743 & 0.037864532 & 0.037801285 \\
\hline $\mathbf{6 8}$ & 0.042565 & 0.042538462 & 0.04149066 & 0.042653588 & 0.042644457 \\
\hline $\mathbf{6 9}$ & 0.047953 & 0.04763233 & 0.046169712 & 0.047771138 & 0.047793744 \\
\hline $\mathbf{7 0}$ & 0.05334 & 0.052941264 & 0.051096575 & 0.053142191 & 0.053143848 \\
\hline $\mathbf{7 1}$ & 0.058728 & 0.058352733 & 0.056234879 & 0.058674178 & 0.058573004 \\
\hline $\mathbf{7 2}$ & 0.064115 & 0.063766034 & 0.061562148 & 0.064273059 & 0.063960975 \\
\hline $\mathbf{7 3}$ & 0.069503 & 0.069103039 & 0.067078961 & 0.069844735 & 0.069211323 \\
\hline $\mathbf{7 4}$ & 0.07489 & 0.074317242 & 0.072816775 & 0.075286964 & 0.074275557 \\
\hline $\mathbf{7 5}$ & 0.080278 & 0.079405903 & 0.078844079 & 0.080489524 & 0.079176406 \\
\hline $\mathbf{7 6}$ & 0.085666 & 0.084430319 & 0.085271074 & 0.085364507 & 0.084027548 \\
\hline $\mathbf{7 7}$ & 0.091053 & 0.089543879 & 0.092253093 & 0.089917917 & 0.089047807 \\
\hline $\mathbf{7 8}$ & 0.096441 & 0.095025345 & 0.099993077 & 0.094352165 & 0.0945695 \\
\hline $\mathbf{7 9}$ & 0.101828 & 0.101313089 & 0.108743143 & 0.099167541 & 0.101042736 \\
\hline & & & & & 8 \\
\hline
\end{tabular}




\begin{tabular}{|c|c|c|c|c|c|}
\hline age(x) & $\begin{array}{c}m_{x} \\
\text { (Female) }\end{array}$ & $\begin{array}{c}m_{x}^{g} \\
(\mathbf{d = 2}, \lambda=\mathbf{1})\end{array}$ & $\begin{array}{c}m_{x}^{g} \\
(\mathbf{d = 2}, \lambda=\mathbf{1 / 1 0})\end{array}$ & $\begin{array}{c}m_{x}^{g} \\
(\mathbf{d = 3}, \lambda=\mathbf{1})\end{array}$ & $\begin{array}{c}m_{x}^{g} \\
(\mathbf{d}=\mathbf{3}, \lambda=\mathbf{1} / \mathbf{1 0})\end{array}$ \\
\hline $\mathbf{8 0}$ & 0.107216 & 0.109038289 & 0.118804925 & 0.105228552 & 0.109038842 \\
\hline $\mathbf{8 1}$ & 0.112603 & 0.119053141 & 0.130527243 & 0.113781129 & 0.119255344 \\
\hline $\mathbf{8 2}$ & 0.117991 & 0.132442012 & 0.144298202 & 0.12642406 & 0.132521756 \\
\hline $\mathbf{8 3}$ & 0.123378 & 0.150472995 & 0.1605267 & 0.145010489 & 0.149801273 \\
\hline $\mathbf{8 4}$ & 0.174944 & 0.174378798 & 0.179605748 & 0.171386204 & 0.172183241 \\
\hline $\mathbf{8 5}$ & 0.22651 & 0.204735987 & 0.201847203 & 0.206907101 & 0.200876312 \\
\hline $\mathbf{8 6}$ & 0.278076 & 0.241911523 & 0.227546619 & 0.252226401 & 0.237263636 \\
\hline $\mathbf{8 7}$ & 0.329642 & 0.286311185 & 0.25702596 & 0.307137705 & 0.282989239 \\
\hline $\mathbf{8 8}$ & 0.381208 & 0.338548725 & 0.290662748 & 0.370515379 & 0.340068701 \\
\hline $\mathbf{8 9}$ & 0.432774 & 0.399556087 & 0.328909457 & 0.440381294 & 0.411025152 \\
\hline $\mathbf{9 0}$ & 0.48434 & 0.470639878 & 0.372305982 & 0.514060224 & 0.4990543 \\
\hline $\mathbf{9 1}$ & 0.535906 & 0.553499841 & 0.421487979 & 0.588367741 & 0.60822515 \\
\hline $\mathbf{9 2}$ & 0.587472 & 0.650232867 & 0.47719374 & 0.659803966 & 0.743727415 \\
\hline $\mathbf{9 3}$ & 0.639038 & 0.763343368 & 0.540271694 & 0.7247598 & 0.912182147 \\
\hline $\mathbf{9 4}$ & 0.690604 & 0.895773045 & 0.611689937 & 0.779749907 & 1.122038246 \\
\hline $\mathbf{9 5}$ & 0.74217 & 1.050954749 & 0.692548518 & 0.821671045 & 1.384084353 \\
\hline $\mathbf{9 6}$ & 0.793736 & 1.232890606 & 0.784094719 & 0.848062906 & 1.71211408 \\
\hline $\mathbf{9 7}$ & 0.845302 & 1.446252547 & 0.88774136 & 0.857335069 & 2.123793534 \\
\hline $\mathbf{9 8}$ & 0.896868 & 1.696503673 & 1.00508817 & 0.848922968 & 2.641794911 \\
\hline $\mathbf{9 9}$ & 0.948434 & 1.990041045 & 1.137946308 & 0.823345771 & 3.295280338 \\
\hline $\mathbf{1 0 0 +}$ & 0.987508 & 2.334362076 & 1.288366276 & 0.782154949 & 4.121847544 \\
\hline & & & & & \\
\hline
\end{tabular}

\section{والشكلين التاليين يوضحين معدلات الوفاة الخام المسواة بالفروض} الأربعة لكلاً من الأكور والإناث: 


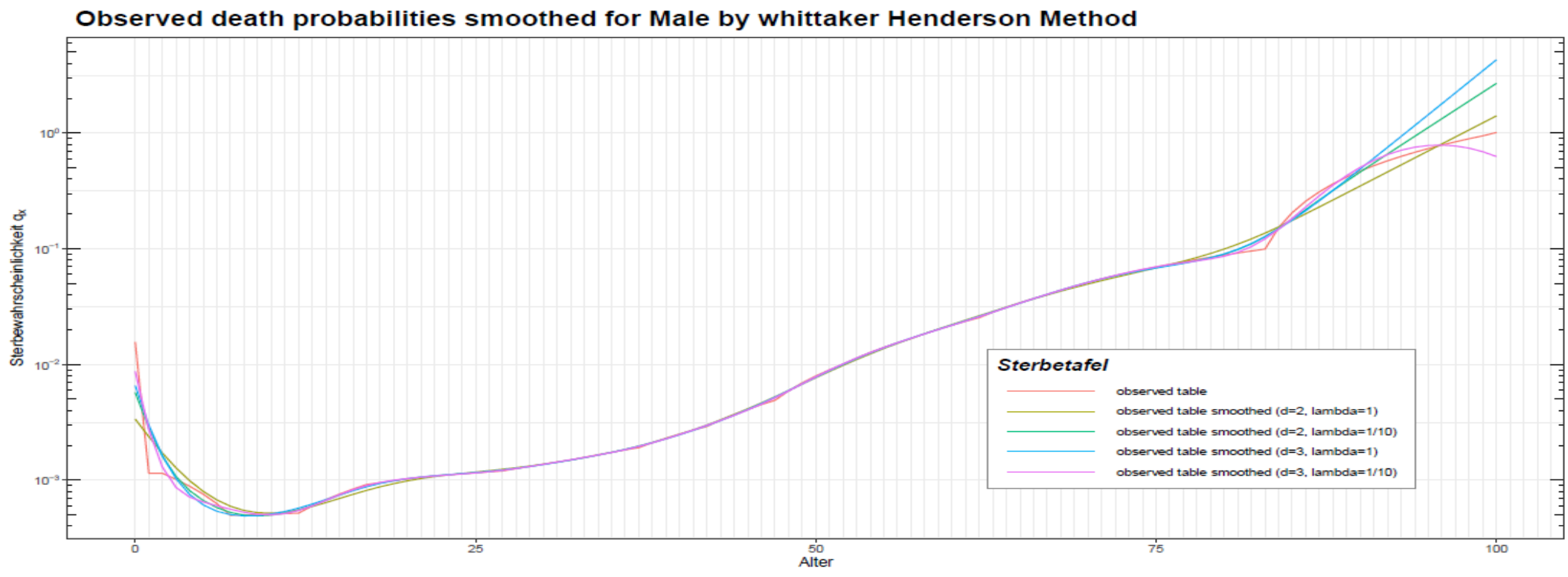




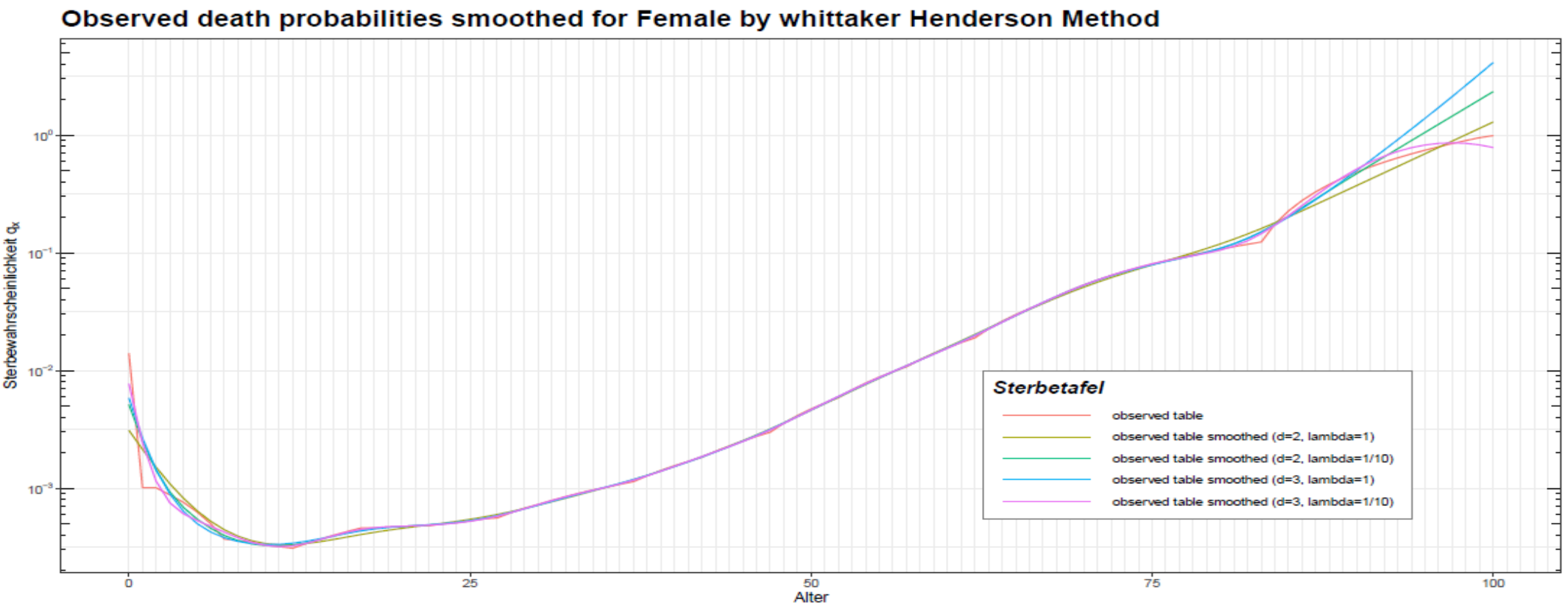




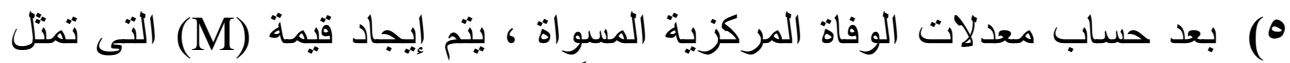
مجموع أقل الفروق من المعادلة التالية لكلاً من الذكور و الإناثاث:

$$
M=F+\lambda S=\sum_{x=0}^{n} W_{x}\left(m_{x}^{g}-m_{x}\right)^{2}+\lambda \sum_{x=0}^{n-d}\left(\Delta^{d} m_{x}^{g}\right)^{2}
$$

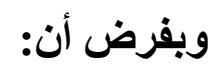

لكل الأعمار $\quad W_{x}=1$

فإن قيمة (M) لكلاً من الذكور والإناث تكون كالتالى :

\begin{tabular}{|c|c|c|c|c|}
\hline & \multicolumn{2}{|c|}{ Male } & \multicolumn{2}{c|}{ Female } \\
\hline$\lambda$ & $\mathrm{d}=2$ & $\mathrm{~d}=3$ & $\mathrm{~d}=2$ & $\mathrm{~d}=3$ \\
\hline 1 & 6.659990626 & 0.269365103 & 4.266836943 & 0.096145367 \\
\hline $1 / 10$ & 0.368690543 & 24.53437927 & 0.226617794 & 6.678123182 \\
\hline
\end{tabular}

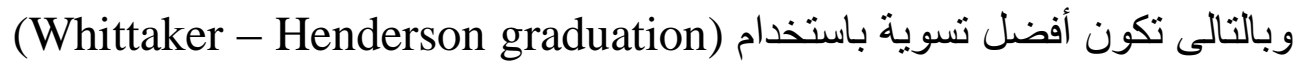

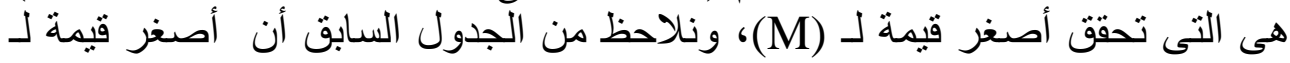

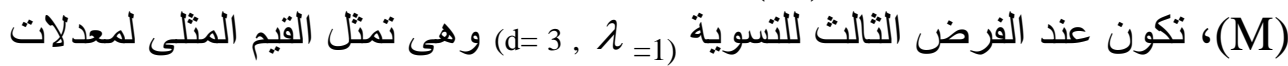

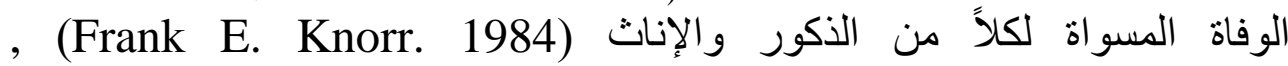
(Siu-Hang Li and Wai-Sum Chan, (Marielynn E. Chanco. 2016) 2004)

$$
\text { نتائج الدراسة: أهم النتائج التى توصلت إليها الدراسة فيما يلى: }
$$

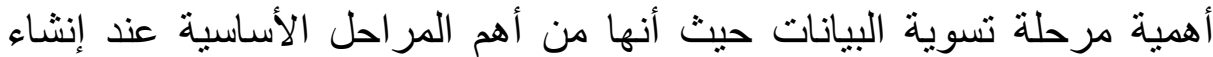

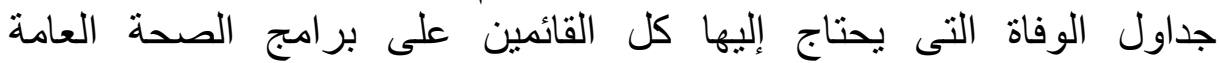
الديموجر افيين، والاكتواريين، والقائمين على تقدير الأقساط بشركات التأمين

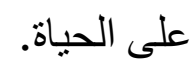

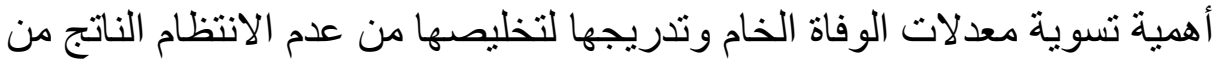
العشوائية فى المقادير المعرضة لاتلة لخطر الوفاة و عدد الوفيات. 
هتخدام الطرق اللامعلمية لتسوية معدلات الوفاة الخام نظراً لأنها لا تشترط

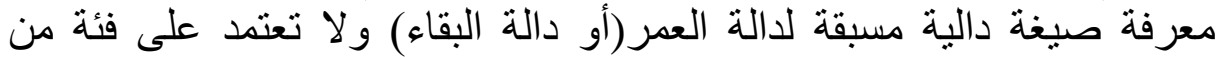

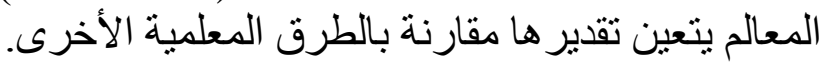

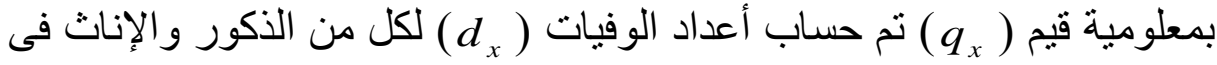

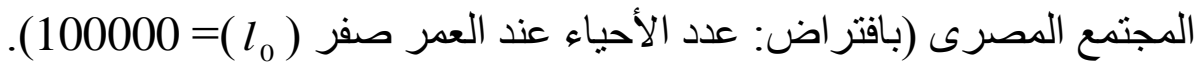

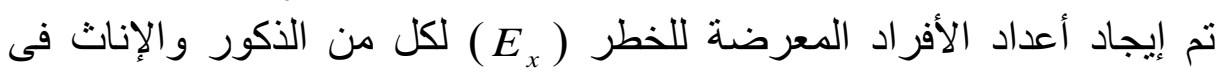

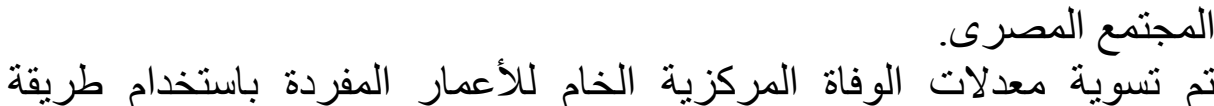
(Whittaker - Henderson graduation) وذلك من خلال أربعة فروض و هم:

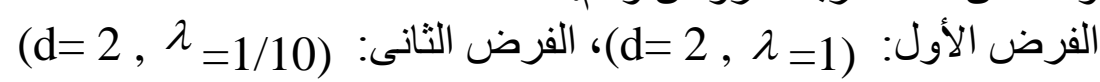

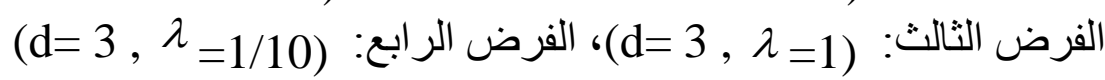

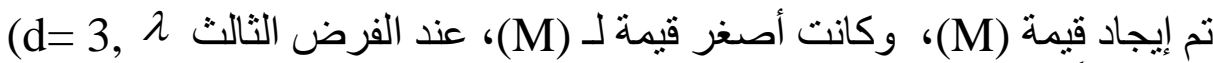

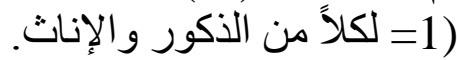

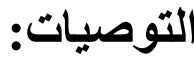
توصى هذه الدر اسة:

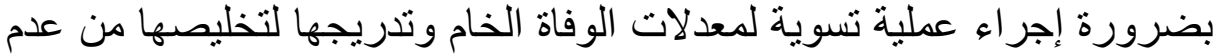

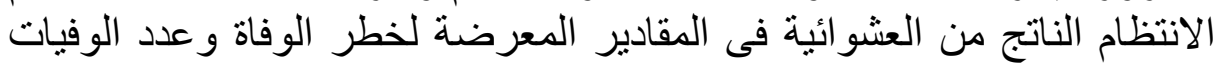

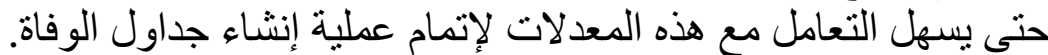

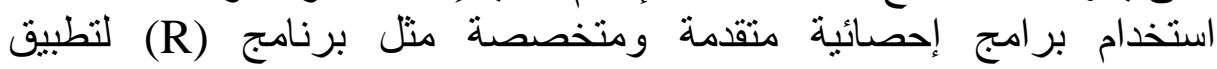
طريقة (Whittaker-Henderson graduation) لتسوية معدلات الوفاة الخام للوصول إلى نتائج أكثر منطقية.

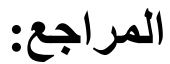

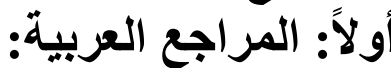

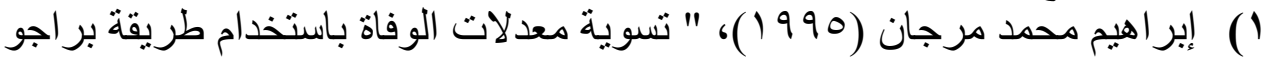

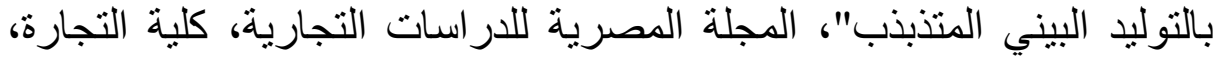

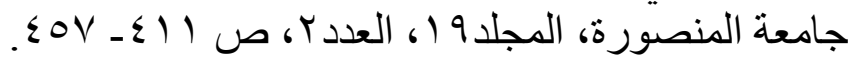




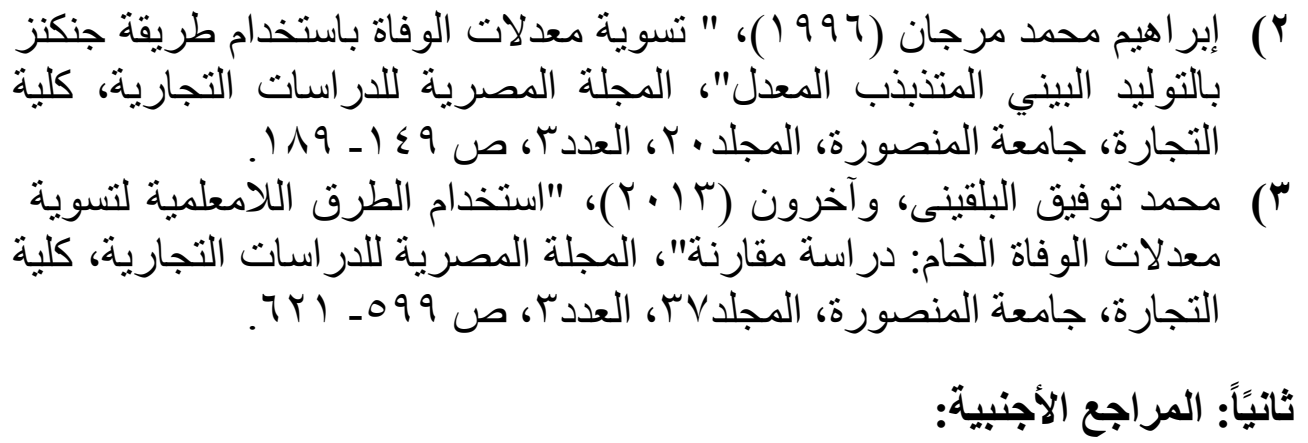

1) Siu-Hang Li and Wai-Sum Chan, (2004). Estimation of complete period life tables for Singaporeans, Journal of Actuarial practice, Vol. 11, 129146

2) Marielynn E. Chanco, (2016). "Mortality Rates Estimation UsingWhittaker-Henderso GraduationTechnique". Social Security System, Quezon City, Philippines, Journal of the Mathematical Society of the Philippines, Vol. 39 Special Issue (2016) pp. 7-16

3) Debon A, Montes F, and Sala R. (2006). "A Comparison of Nonparametric Methods in the Graduation of Mortality: Application to Data from the Valencia Region (Spain)". International Statistical Review, Vol 74, 2, pp.215-233.

4) Frank E. Knorr. (1984). "Multidimensional Whittaker- Henderson graduation". Transactions of Society of actuaries International Statistical, Vol 36, pp.213-255.

5) Takis Papaioannou and Athanasios Sachlas. (2004). "Graduation of mortality rates revisited". University of Piraeus.

6) London, D. (1985). Graduation: The Revision of Estimates, ACTEX Publications, Winsted, Connecticut.

7) Walter B. Lowrie. (1982)" An Extension of the Whittaker-Henderson Method of Graduation", Transactions of Society of Actuaries, Vol. 34, pp. 329-372.

8) Johnny Siu-Hang Li, Mary R. Hardy, and Ken Seng Tan. (2008)" Threshold Life Tables and Their Applications ", North American Actuarial Journal, Vol. 12, Number 2, Published online: 28 Dec 2012, pp. 99-115. 
9) Whittaker. mortality Table .R, Smooth a life table using the WhittakerHenderson method

10) Miller M.D. (1946). Elements of Graduation. New York: Actuarial Society of America and American Institute of Actuaries.

11) Reinhold Kainhofer (2018). R package A Framework for various Types of Mortality/ Life Tables (Mortality Tables). 


\section{ملحق (1)}

جدول (1): جدول الحياة الكامل لجمهورية مصر العربية وفقاً لتعداد السكان لعام ( r. IV

\begin{tabular}{|c|c|c|c|c|c|c|c|}
\hline Age & $\boldsymbol{q}_{\boldsymbol{x}}$ & Age & $\boldsymbol{q}_{\boldsymbol{x}}$ & Age & $\boldsymbol{q}_{\boldsymbol{x}}$ & Age & $\boldsymbol{q}_{\boldsymbol{x}}$ \\
\hline 0 & 0.015517417 & 26 & 0.001177265 & 52 & 0.009946667 & 78 & 0.077673451 \\
\hline 1 & 0.001144224 & 27 & 0.001199207 & 53 & 0.011280118 & 79 & 0.081083734 \\
\hline 2 & 0.001144224 & 28 & 0.001257816 & 54 & 0.012611783 & 80 & 0.084481939 \\
\hline 3 & 0.001014546 & 29 & 0.001316421 & 55 & 0.013941666 & 81 & 0.087868129 \\
\hline 4 & 0.000884851 & 30 & 0.001375023 & 56 & 0.015269771 & 82 & 0.091242368 \\
\hline 5 & 0.000755139 & 31 & 0.001433622 & 57 & 0.0165961 & 83 & 0.09460472 \\
\hline 6 & 0.00062541 & 32 & 0.001492217 & 58 & 0.018282228 & 84 & 0.141509357 \\
\hline 7 & 0.000495664 & 33 & 0.001574882 & 59 & 0.019965492 & 85 & 0.186160196 \\
\hline 8 & 0.000500093 & 34 & 0.001657541 & 60 & 0.021645898 & 86 & 0.22871587 \\
\hline 9 & 0.000504522 & 35 & 0.001740192 & 61 & 0.023323455 & 87 & 0.269320468 \\
\hline 10 & 0.000508951 & 36 & 0.001822837 & 62 & 0.024998169 & 88 & 0.308105159 \\
\hline 11 & 0.00051338 & 37 & 0.001905475 & 63 & 0.02788418 & 89 & 0.345189616 \\
\hline 12 & 0.000517809 & 38 & 0.002104904 & 64 & 0.030761768 & 90 & 0.380683241 \\
\hline 13 & 0.000597493 & 39 & 0.002304292 & 65 & 0.033630971 & 91 & 0.414686251 \\
\hline 14 & 0.000677171 & 40 & 0.002503641 & 66 & 0.036491826 & 92 & 0.447290614 \\
\hline 15 & 0.000756843 & 41 & 0.00270295 & 67 & 0.039344368 & 93 & 0.478580887 \\
\hline 16 & 0.000836508 & 42 & 0.002902219 & 68 & 0.042891876 & 94 & 0.508634947 \\
\hline 17 & 0.000916167 & 43 & 0.003298364 & 69 & 0.04642657 & 95 & 0.537524634 \\
\hline 18 & 0.000950834 & 44 & 0.003694352 & 70 & 0.049948519 & 96 & 0.565316329 \\
\hline 19 & 0.000985501 & 45 & 0.004090182 & 71 & 0.053457792 & 97 & 0.59207146 \\
\hline 20 & 0.001020166 & 46 & 0.004485856 & 72 & 0.056954457 & 98 & 0.617846958 \\
\hline 21 & 0.00105483 & 47 & 0.004881373 & 73 & 0.060438583 & 99 & 0.642695659 \\
\hline 22 & 0.001089493 & 48 & 0.005896493 & 74 & 0.063910236 & 100 & 0.671795336 \\
\hline 23 & 0.001111436 & 49 & 0.006910582 & 75 & 0.067369483 & & \\
\hline 24 & 0.00113338 & 50 & 0.007923639 & 76 & 0.07081639 & & \\
\hline 25 & 0.001155323 & 51 & 0.008935667 & 77 & 0.074251025 & & \\
\hline & & & & & & & \\
\hline
\end{tabular}


جدول (ץ): جدول الحياة الكامل لجمهورية مصر العربية وفقاً لتعداد السكان لعام

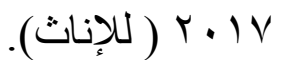

\begin{tabular}{|c|c|c|c|c|c|c|c|}
\hline Age & $\boldsymbol{q}_{\boldsymbol{x}}$ & Age & $\boldsymbol{q}_{\boldsymbol{x}}$ & Age & $\boldsymbol{q}_{\boldsymbol{x}}$ & Age & $\boldsymbol{q}_{\boldsymbol{x}}$ \\
\hline 0 & 0.01393 & 26 & 0.00055 & 52 & 0.00586 & 78 & 0.09200 \\
\hline 1 & 0.00101 & 27 & 0.00056 & 53 & 0.00683 & 79 & 0.09689 \\
\hline 2 & 0.00101 & 28 & 0.00062 & 54 & 0.00779 & 80 & 0.10176 \\
\hline 3 & 0.00088 & 29 & 0.00068 & 55 & 0.00876 & 81 & 0.10660 \\
\hline 4 & 0.00076 & 30 & 0.00073 & 56 & 0.00972 & 82 & 0.11142 \\
\hline 5 & 0.00063 & 31 & 0.00079 & 57 & 0.01069 & 83 & 0.11620959 \\
\hline 6 & 0.00050 & 32 & 0.00085 & 58 & 0.01230 & 84 & 0.160872563 \\
\hline 7 & 0.00037 & 33 & 0.00091 & 59 & 0.01391 & 85 & 0.203466747 \\
\hline 8 & 0.00036 & 34 & 0.00097 & 60 & 0.01552 & 86 & 0.244132628 \\
\hline 9 & 0.00035 & 35 & 0.00102 & 61 & 0.01712 & 87 & 0.282998254 \\
\hline 10 & 0.00033 & 36 & 0.00108 & 62 & 0.01872 & 88 & 0.32018058 \\
\hline 11 & 0.00032 & 37 & 0.00114 & 63 & 0.02230 & 89 & 0.355786646 \\
\hline 12 & 0.00031 & 38 & 0.00128 & 64 & 0.02587 & 90 & 0.389914603 \\
\hline 13 & 0.00034 & 39 & 0.00142 & 65 & 0.02943 & 91 & 0.422654622 \\
\hline 14 & 0.00037 & 40 & 0.00155 & 66 & 0.03297 & 92 & 0.454089684 \\
\hline 15 & 0.00040 & 41 & 0.00169 & 67 & 0.03650 & 93 & 0.484296283 \\
\hline 16 & 0.00043 & 42 & 0.00183 & 68 & 0.04168 & 94 & 0.513345051 \\
\hline 17 & 0.00046 & 43 & 0.00206 & 69 & 0.04683 & 95 & 0.541301306 \\
\hline 18 & 0.00046 & 44 & 0.00229 & 70 & 0.05195 & 96 & 0.568225544 \\
\hline 19 & 0.00047 & 45 & 0.00252 & 71 & 0.05705 & 97 & 0.594173876 \\
\hline 20 & 0.00047 & 46 & 0.00275 & 72 & 0.06212 & 98 & 0.619198416 \\
\hline 21 & 0.00048 & 47 & 0.00299 & 73 & 0.06717 & 99 & 0.643347634 \\
\hline 22 & 0.00048 & 48 & 0.00356 & 74 & 0.07219 & 100 & 0.661091241 \\
\hline 23 & 0.00050 & 49 & 0.00414 & 75 & 0.07718 & & \\
\hline 24 & 0.00051 & 50 & 0.00471 & 76 & 0.08215 & & \\
\hline 25 & 0.00053 & 51 & 0.00529 & 77 & 0.08709 & & \\
\hline & & & & & & & \\
\hline
\end{tabular}

